\title{
The ALMA Spectroscopic Survey in the HUDF: CO Luminosity Functions and the Molecular Gas Content of Galaxies through Cosmic History
}

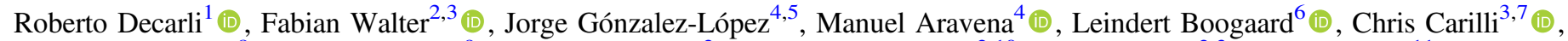
Pierre $\operatorname{Cox}^{8}$, Emanuele Daddi ${ }^{9}$ (1), Gergö Popping ${ }^{2}$ (1), Dominik Riechers ${ }^{2,10}$ (10), Bade Uzgil ${ }^{2,3}$ (1), Axel Weiss ${ }^{11}$ (1),

Roberto J. Assef ${ }^{4}$ (1) , Roland Bacon ${ }^{12}$, Franz Erik Bauer ${ }^{5,13,14}$ (1) , Frank Bertoldi ${ }^{15}$ (10), Rychard Bouwens ${ }^{5}$ (i), Thierry Contini ${ }^{16}$ (1), Paulo C. Cortes ${ }^{17,18}$ (1) , Elisabete da Cunha ${ }^{19}$, Tanio Díaz-Santos ${ }^{4}$, David Elbaz ${ }^{8}$, Hanae Inami ${ }^{11,20}$, Jacqueline Hodge ${ }^{5}$ (1), Rob Ivison ${ }^{21,22}$ (1), Olivier Le Fèvre ${ }^{23}$, Benjamin Magnelli ${ }^{15}$ (1), Mladen Novak ${ }^{2}$ (i), Pascal Oesch ${ }^{24,31}$ (i) , Hans-Walter Rix ${ }^{2}$ (i) (i), Mark T. Sargent ${ }^{25}$ (1), Ian Smail ${ }^{26}$ (1), A. Mark Swinbank ${ }^{27}$ (1) , Rachel S. Somerville ${ }^{27,28}$, Paul van der Werf ${ }^{5}$, Jeff Wagg ${ }^{29}$, and Lutz Wisotzki ${ }^{30}$

${ }^{1}$ INAF-Osservatorio di Astrofisica e Scienza dello Spazio, via Gobetti 93/3, I-40129, Bologna, Italy; roberto.decarli@inaf.it

${ }^{2}$ Max Planck Institut für Astronomie, Königstuhl 17, D-69117 Heidelberg, Germany

${ }^{3}$ National Radio Astronomy Observatory, Pete V. Domenici Array Science Center, P.O. Box O, Socorro, NM 87801, USA

${ }^{4}$ Núcleo de Astronomía, Facultad de Ingeniería y Ciencias, Universidad Diego Portales, Av. Ejército 441, Santiago, Chile

${ }^{5}$ Instituto de Astrofísica, Facultad de Física, Pontificia Universidad Católica de Chile Av. Vicuña Mackenna 4860, 782-0436 Macul, Santiago, Chile ${ }^{6}$ Leiden Observatory, Leiden University, P.O. Box 9513, NL-2300 RA Leiden, The Netherlands

${ }^{7}$ Battcock Centre for Experimental Astrophysics, Cavendish Laboratory, Cambridge CB3 OHE, UK

${ }^{8}$ Institut d'Astrophysique de Paris, Sorbonne Université, CNRS, UMR 7095, 98 bis bd Arago, F-7014 Paris, France

${ }^{9}$ Laboratoire AIM, CEA/DSM-CNRS-Universite Paris Diderot, Irfu/Service d'Astrophysique, CEA Saclay, Orme des Merisiers, F-91191 Gif-sur-Yvette cedex, France

${ }^{10}$ Cornell University, 220 Space Sciences Building, Ithaca, NY 14853, USA

${ }^{11}$ Max-Planck-Institut für Radioastronomie, Auf dem Hügel 69, D-53121 Bonn, Germany

${ }^{12}$ Univ. Lyon 1, ENS de Lyon, CNRS, Centre de Recherche Astrophysique de Lyon (CRAL) UMR5574, F-69230 Saint-Genis-Laval, France

${ }^{13}$ Millennium Institute of Astrophysics (MAS), Nuncio Monseñor Sótero Sanz 100, Providencia, Santiago, Chile

${ }^{14}$ Space Science Institute, 4750 Walnut Street, Suite 205, Boulder, CO 80301, USA

${ }^{15}$ Argelander-Institut für Astronomie, Universität Bonn, Auf dem Hügel 71, D-53121 Bonn, Germany

${ }^{16}$ Institut de Recherche en Astrophysique et Planétologie (IRAP), Université de Toulouse, CNRS, UPS, F-31400 Toulouse, France

${ }^{17}$ Joint ALMA Observatory-ESO, Av. Alonso de Córdova, 3104, Santiago, Chile

${ }^{18}$ National Radio Astronomy Observatory, 520 Edgemont Road, Charlottesville, VA 22903, USA

${ }^{19}$ Research School of Astronomy and Astrophysics, Australian National University, Canberra, ACT 2611, Australia

${ }^{20}$ Hiroshima Astrophysical Science Center, Hiroshima University, 1-3-1 Kagamiyama, Higashi-Hiroshima, Hiroshima, 739-8526, Japan

${ }^{21}$ European Southern Observatory, Karl-Schwarzschild-Strasse 2, D-85748, Garching, Germany

${ }^{22}$ Institute for Astronomy, University of Edinburgh, Royal Observatory, Blackford Hill, Edinburgh EH9 3HJ, UK

${ }^{23}$ Aix Marseille Université, CNRS, LAM (Laboratoire d'Astrophysique de Marseille), UMR 7326, F-13388 Marseille, France

${ }^{24}$ Department of Astronomy, University of Geneva, Ch. des Maillettes 51, 1290 Versoix, Switzerland

${ }^{25}$ Astronomy Centre, Department of Physics and Astronomy, University of Sussex, Brighton, BN1 9QH, UK

${ }^{26}$ Centre for Extragalactic Astronomy, Department of Physics, Durham University, South Road, Durham, DH1 3LE, UK

${ }^{27}$ Department of Physics and Astronomy, Rutgers, The State University of New Jersey, 136 Frelinghuysen Road, Piscataway, NJ 08854, USA

${ }^{28}$ Center for Computational Astrophysics, Flatiron Institute, 162 5th Avenue, New York, NY 10010, USA

${ }^{29}$ SKA Organization, Lower Withington Macclesfield, Cheshire SK11 9DL, UK

${ }^{30}$ Leibniz-Institut für Astrophysik Potsdam, An der Sternwarte 16, D-14482 Potsdam, Germany

${ }^{31}$ International Associate, Cosmic Dawn Center (DAWN) at the Niels Bohr Institute, University of Copenhagen and DTU-Space, Technical University of Denmark, Copenhagen, Denmark

Received 2018 December 21; revised 2019 March 17; accepted 2019 April 3; published 2019 September 11

\begin{abstract}
We use the results from the ALMA large program ASPECS, the spectroscopic survey in the Hubble Ultra Deep Field (HUDF), to constrain CO luminosity functions of galaxies and the resulting redshift evolution of $\rho\left(\mathrm{H}_{2}\right)$. The broad frequency range covered enables us to identify $\mathrm{CO}$ emission lines of different rotational transitions in the HUDF at $z>1$. We find strong evidence that the CO luminosity function evolves with redshift, with the knee of the $\mathrm{CO}$ luminosity function decreasing in luminosity by an order of magnitude from $\sim 2$ to the local universe. Based on Schechter fits, we estimate that our observations recover the majority (up to $\sim 90 \%$, depending on the assumptions on the faint end) of the total cosmic CO luminosity at $z=1.0-3.1$. After correcting for $\mathrm{CO}$ excitation, and adopting a Galactic CO-to- $\mathrm{H}_{2}$ conversion factor, we constrain the evolution of the cosmic molecular gas density $\rho\left(\mathrm{H}_{2}\right)$ : this cosmic gas density peaks at $z \sim 1.5$ and drops by a factor of $6.5_{-1.4}^{+1.8}$ to the value measured locally. The observed evolution in $\rho\left(\mathrm{H}_{2}\right)$, therefore, closely matches the evolution of the cosmic star formation rate density $\rho_{\text {SFR }}$. We verify the robustness of our result with respect to assumptions on source inclusion and/or CO excitation. As the cosmic star formation history can be expressed as the product of the star formation efficiency and the cosmic density of molecular gas, the similar evolution of $\rho\left(\mathrm{H}_{2}\right)$ and $\rho_{\mathrm{SFR}}$ leaves only little room for a significant evolution of the average star formation efficiency in galaxies since $z \sim 3$ (85\% of cosmic history).
\end{abstract}

Key words: galaxies: evolution - galaxies: high-redshift - galaxies: ISM - galaxies: luminosity function, mass function - surveys

Supporting material: machine-readable table 


\section{Introduction}

The molecular phase of the interstellar medium (ISM) is the birthplace of stars, and therefore it plays a central role in the evolution of galaxies (see the reviews in Kennicutt \& Evans 2012; Bolatto et al. 2013; Carilli \& Walter 2013). The cosmic history of star formation (see, e.g., Madau \& Dickinson 2014), i.e., the mass of stars formed per unit time in a cosmological volume (or cosmic star formation rate density, $\left.\rho_{\mathrm{SFR}}\right)$ throughout cosmic time, increased from early cosmic epochs up to a peak at $z=1-3$, and then declined by a factor of $\sim 8$ until the present day. This could be explained by a larger supply of molecular gas (the fuel for star formation) in high- $z$ galaxies; by physical properties of the gas, that could more efficiently form stars; or by a combination of both. The characterization of the content and properties of the molecular ISM in galaxies at different cosmic epochs is therefore fundamental to our understanding of galaxy formation and evolution.

The $\mathrm{H}_{2}$ molecule, the main constituent of molecular gas, is a poor radiator: it lacks rotational transitions, and the energy levels of vibrational lines are populated significantly only at relatively high temperatures $\left(T_{\mathrm{ex}}>500 \mathrm{~K}\right)$ that are not typical of the cold, star-forming ISM (Omont 2007). On the other hand, the carbon monoxide molecule, ${ }^{12} \mathrm{CO}$ (hereafter, $\mathrm{CO}$ ) is the second most abundant molecule in the universe. Thanks to its bright rotational transitions, it has been detected even at the highest redshifts $(z \sim 7$; e.g., Riechers et al. 2013; Strandet et al. 2017; Venemans et al. 2017; Marrone et al. 2018). Redshifted CO lines are observed in the radio and millimeter $(\mathrm{mm})$ transparent windows of the atmosphere, thus becoming accessible to facilities such as the Jansky Very Large Array (JVLA), the IRAM NOrthern Expanded Millimeter Array (NOEMA), and the Atacama Large Millimeter Array (ALMA). $\mathrm{CO}$ is therefore the preferred observational probe of the molecular gas content in galaxies at high redshift.

To date, more than 250 galaxies have been detected in $\mathrm{CO}$ at $z>1$, the majority of which are quasar host galaxies or submillimeter galaxies (see Carilli \& Walter 2013 for a review); gravitationally lensed galaxies (e.g., Riechers et al. 2010; Harris et al. 2012; Dessauges-Zavadsky et al. 2015; Aravena et al. 2016c; Dessauges-Zavadsky et al. 2017; González-López et al. 2017); and (proto)clusters of galaxies (e.g., Aravena et al. 2012; Chapman et al. 2015; Seko et al. 2016; Hayatsu et al. 2017; Lee et al. 2017; Rudnick et al. 2017; Hayashi et al. 2018; Miller et al. 2018; Oteo et al. 2018). The remainder are galaxies selected based on their stellar mass $\left(M_{*}\right)$, star formation rate $(\mathrm{SFR})$, and/or optical/near-infrared colors (e.g., Daddi et al. 2010a, 2010b; Genzel et al. 2010, 2011, 2015; Tacconi et al. 2010, 2013, 2018; Pappovich et al. 2016). These studies were instrumental in shaping our understanding of the interplay between molecular gas reservoirs and star formation in massive $z>1$ galaxies on and above the "main sequence" of star-forming galaxies (Noeske et al. 2007; Elbaz et al. 2011). For example, these galaxies are found to have high molecular gas fractions $M_{\mathrm{H} 2} / M_{*}$ compared to galaxies in the local universe. The depletion time, $t_{\mathrm{dep}}=M_{\mathrm{H} 2} / \mathrm{SFR}$, i.e., the time required to consume the entire molecular gas content of a galaxy at the present rate of star formation, is shorter in starburst galaxies than in galaxies on the main sequence (see, e.g., Silverman et al. 2015, 2018; Schinnerer et al. 2016; Scoville et al. 2017; Tacconi et al. 2018). However, by nature these targeted studies are potentially biased toward specific types of galaxies (e.g., massive, star-forming galaxies), and consequently might fail to capture the full diversity of gas-rich galaxies in the universe.

Spectral line scans provide a complementary approach. These are interferometric observations over wide frequency ranges, targeting "blank" regions of the sky. Gas, traced mainly via CO lines, is searched for at any position and frequency, without preselection based on other wavelengths. This provides us with a flux-limited census of the gas content in well-defined cosmological volumes. The first molecular scan reaching sufficient depth to detect MS galaxies targeted a $\sim 1 \operatorname{arcmin}^{2}$ region in the Hubble Deep Field North (HDF-N; Williams et al. 1996) using the IRAM Plateau de Bure Interferometer (PdBI; see Decarli et al. 2014). The scan resulted in the first redshift measurement for the archetypal submillimeter galaxy $\operatorname{HDF} 850.1(z=5.183$, see Walter et al. 2012), and in the discovery of massive $\left(>10^{10} M_{\odot}\right)$ gaseous reservoirs associated with galaxies at $z \sim 2$, including one with no obvious optical/NIR counterpart (Decarli et al. 2014). These observations enabled the first, admittedly loose constraints on the $\mathrm{CO}$ luminosity functions (LFs) and on the cosmic density of molecular gas in galaxies, $\rho\left(\mathrm{H}_{2}\right)$, as a function of redshift (Walter et al. 2014). The HDF-N was also part of a second large observing campaign using the JVLA, the COLDz project. This effort ( $>300 \mathrm{hr}$ of observations) targeted a $\sim 48 \operatorname{arcmin}^{2}$ area in the GOODS-North footprint (Giavalisco et al. 2004), and a $\sim 8 \operatorname{arcmin}^{2}$ region in COSMOS (Scoville et al. 2007), sampling the frequency range $30-38 \mathrm{GHz}$ (Lentati et al. 2015; Pavesi et al. 2018). This exposed the $\mathrm{CO}(1-0)$ emission in galaxies at $z \approx 2.0-2.8$ and the $\mathrm{CO}(2-1)$ emission at $z \approx 4.9-6.7$. The unprecedentedly large area covered by COLDz resulted in the best constraints on the CO LFs at $z>2$ so far, especially at the bright end (Riechers et al. 2019).

In ALMA Cycle 2, we scanned the $3 \mathrm{~mm}$ and $1.2 \mathrm{~mm}$ windows $(84-115 \mathrm{GHz}$ and $212-272 \mathrm{GHz}$, respectively) in a $\sim 1 \operatorname{arcmin}^{2}$ region in the Hubble Ultra Deep Field (HUDF; Beckwith et al. 2006). This pilot program, dubbed the ALMA Spectroscopic Survey in the HUDF (ASPECS; Walter et al. 2016), pushed the constraints on the CO LFs at high redshift toward the expected knee of the CO LFs (Decarli et al. 2016a). By capitalizing on the combination of the $3 \mathrm{~mm}$ and $1.2 \mathrm{~mm}$ data, and on the unparalleled wealth of ancillary information available in the HUDF, Decarli et al. (2016b) were able to measure $\mathrm{CO}$ excitation in some of the observed sources, and to relate the $\mathrm{CO}$ emission to other properties of the observed galaxies at various wavelengths. Furthermore, the collapsed $1.2 \mathrm{~mm}$ data cube resulted in the deepest dust continuum image ever obtained at these wavelengths $\left(\sigma=13 \mu \mathrm{Jy}\right.$ beam $\left.^{-1}\right)$, which allowed us to resolve $\sim 80 \%$ of the cosmic infrared background (Aravena et al. 2016a). The $1.2 \mathrm{~mm}$ data were also exploited to perform a systematic search for [C II] emitters at $z=6-8$ (Aravena et al. 2016b), as well as to constrain the IRX $-\beta$ relation at high redshift (Bouwens et al. 2016). Finally, the ASPECS Pilot provided first direct measurements of the impact of foreground $\mathrm{CO}$ lines on measurements of the cosmic microwave background fluctuations, which is critical for intensity mapping experiments (Carilli et al. 2016).

The ASPECS Pilot program was limited by the small area surveyed. Here we present results from the ASPECS Large Program (ASPECS LP). The project replicates the survey strategy of the ASPECS Pilot, but on a larger mosaic that covers most of the Hubble eXtremely Deep Field (XDF), the region of the HUDF where the deepest near-infrared data are available (Illingworth et al. 2013; Koekemoer et al. 2013; see 


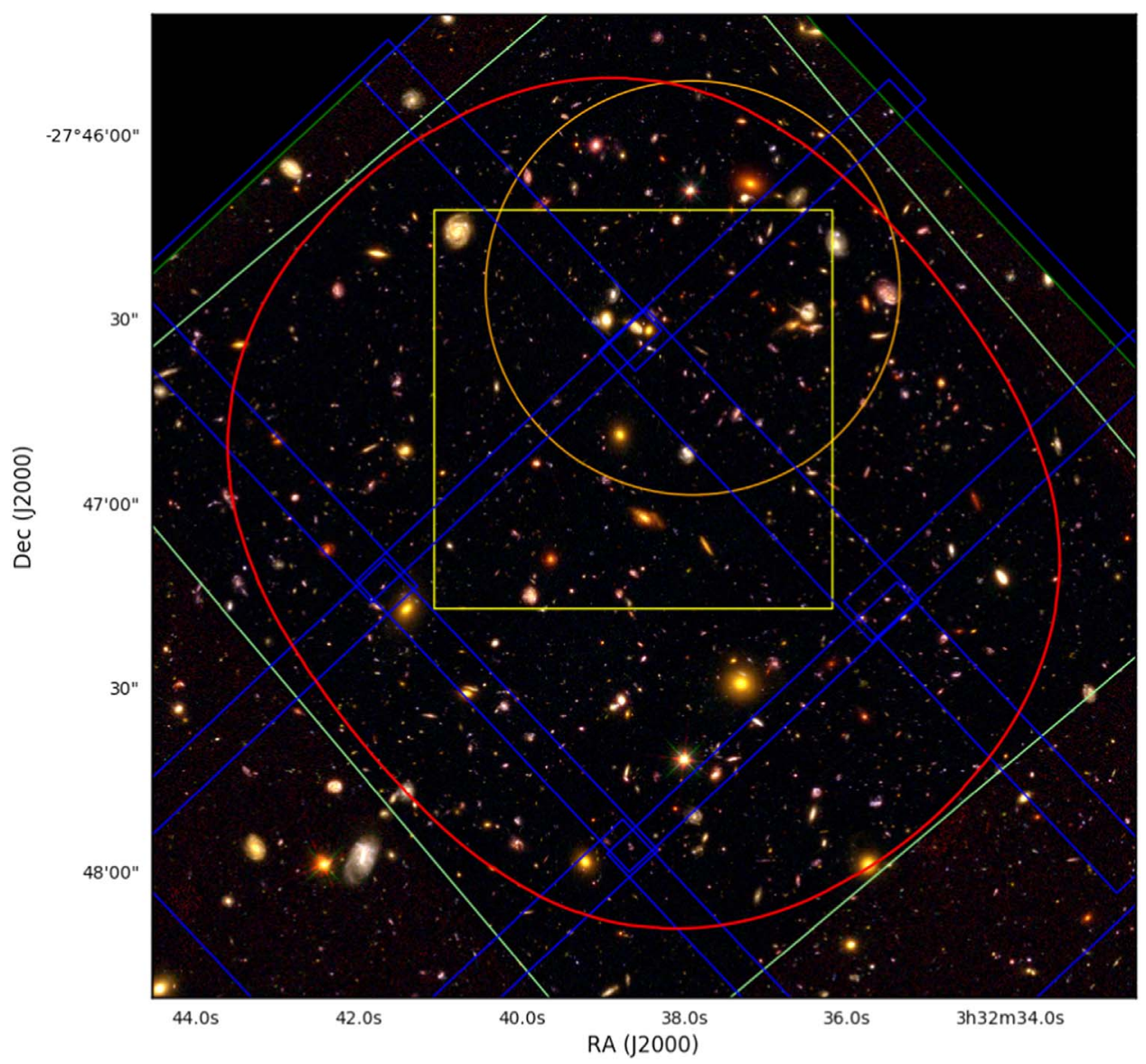

Figure 1. Hubble RGB images (red: F105W filter, green: F770W filter, and blue: F435W filter) of the Hubble Ultra Deep Field (dark green contour). For comparison, we plot the coverage of the Hubble eXtremely Deep Field (XDF; Illingworth et al. 2013; Koekemoer et al. 2013) in light green; the pointings of the MUSE UDF survey (Bacon et al. 2017) in blue; and the deep MUSE pointing (Bacon et al. 2017) in yellow. The 50\% sensitivity contours of the ASPECS pilot (Walter et al. 2016) and of the ASPECS LP $3 \mathrm{~mm}$ survey are shown in orange and red, respectively (see also González-López et al. 2019). The area covered in our study encompasses $>7000$ cataloged galaxies, with hundreds of spectroscopic redshifts, and photometry in $>30$ bands.

Figure 1). Here we present and focus on the ASPECS LP $3 \mathrm{~mm}$ data, which have been collected in ALMA Cycle 4. We discuss the survey strategy and observations, the data reduction, and the ancillary data set, and we use the $\mathrm{CO}$ detections from the $3 \mathrm{~mm}$ data to measure the CO LFs in various redshift bins, and to infer the cosmic gas density $\rho\left(\mathrm{H}_{2}\right)$ as a function of redshift. In González-López et al. (2019; hereafter, GL19), we present our search for line and continuum sources, and assess their reliability and completeness. Aravena et al. (2019) place the ASPECS LP $3 \mathrm{~mm}$ results in the context of the main-sequence narrative. Boogaard et al. (2019) capitalize on the sensitive VLT/ MUSE Integral Field Spectroscopy of the field, in order to address how our $\mathrm{CO}$ detections relate with the properties of the galaxies as inferred from rest-frame optical/UV wavelengths. Finally, Popping et al. (2019) compare the ASPECS LP $3 \mathrm{~mm}$ results to state-of-the-art predictions from cosmological simulations and semianalytical models.

The structure of this paper is as follows. In Section 2, we present the survey strategy, the observations, and the data reduction. In Section 3 we summarize the ancillary information available for the galaxies in this field. In Section 4 we present the main results of this study, and in Section 5 we discuss our findings and compare them with similar works in the literature. Finally, in Section 6 we infer our conclusions.
Throughout this paper we adopt a $\Lambda$ CDM cosmological model with $H_{0}=70 \mathrm{~km} \mathrm{~s}^{-1} \mathrm{Mpc}^{-1}, \Omega_{\mathrm{m}}=0.3$, and $\Omega_{\Lambda}=$ 0.7 (consistent with the measurements by the Planck Collaboration et al. 2016). Magnitudes are reported in the AB photometric system. For consistency with the majority of the literature on this field, in our analysis, we adopt a Chabrier (2003) stellar initial mass function.

\section{Observations and Data Processing}

\subsection{Survey Design and Observations}

The ASPECS LP survey consists of a $150 \mathrm{hr}$ program in ALMA Cycle 4 (Program ID: 2016.1.00324.L). ASPECS LP comprises two scans, at $3 \mathrm{~mm}$ and $1.2 \mathrm{~mm}$. The $3 \mathrm{~mm}$ survey presented here took $68 \mathrm{hr}$ of telescope time (including calibrations and overheads), and was executed between 2016 December 2-21 (ALMA Cycle 4).

These observations comprised 17 pointings covering most of the XDF (Illingworth et al. 2013; Koekemoer et al. 2013; see Figure 1). The pointings were arranged in a hexagonal pattern, distanced by 26 ". 4 (the half-width of the primary beam of ALMA $12 \mathrm{~m}$ antennas at the high-frequency end of ALMA band 3), thus ensuring Nyquist sampling and spatially homogeneous noise in the mosaic. For reference, the central pointing is centered at R.A. $=03: 32: 38.5$ and decl. $=-27: 47: 00$ (J2000.0). The total 


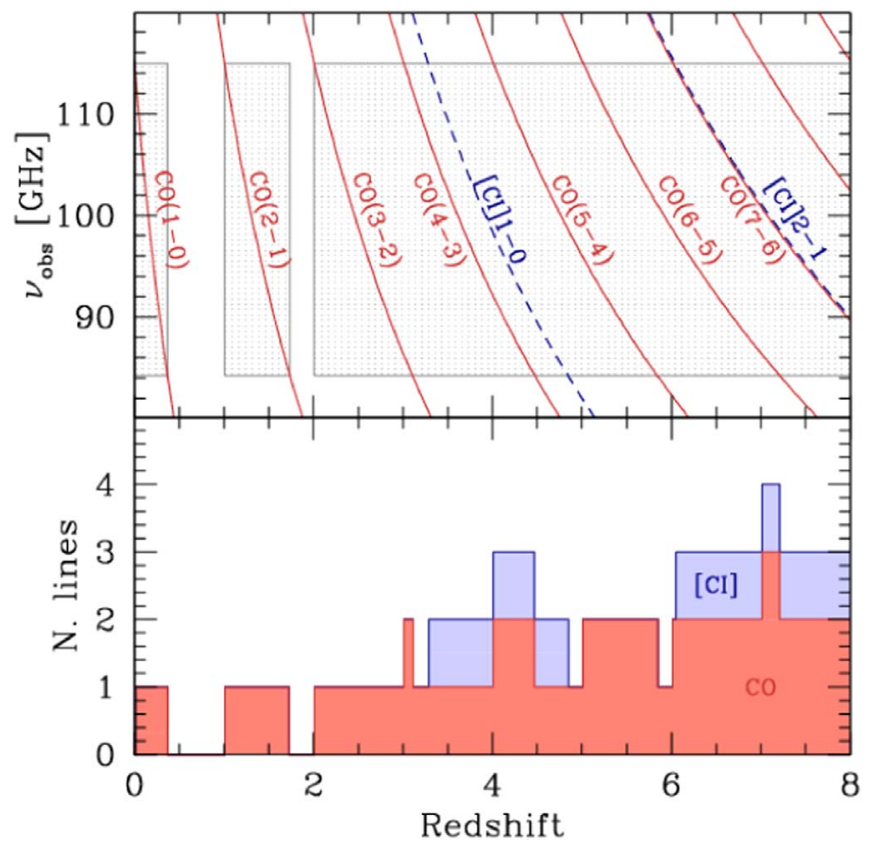

Figure 2. Top: the observed frequency of various $\mathrm{CO}$ and $[\mathrm{C} \mathrm{I}]$ transitions covered in our $3 \mathrm{~mm}$ scan, as a function of redshift. The shaded area marks the parameter space sampled in our study. Bottom: number of $\mathrm{CO}$ or $[\mathrm{C} \mathrm{I}]$ transitions observable in our $3 \mathrm{~mm}$ scan (exclusively based on frequency coverage), as a function of redshift. The frequency range encompassed in our study enables the detection of CO at $z \lesssim 0.37,1.0 \lesssim z \lesssim 1.7$, and virtually at any $z \gtrsim 2.00$. Additionally, our scan covers 2 or more transitions at most redshifts above $z \sim 3$.

area covered at the center of the frequency scan $(\approx 99.5 \mathrm{GHz})$ with primary beam attenuation $<0.5$ is $4.6 \operatorname{arcmin}^{2}$. The observing strategy capitalized on the fast slew of the ALMA antennas in order to fully cover the entire mosaic between each phase calibrator observation. The survey was executed with the array in a relatively compact (C40-3) configuration. Baselines ranged between 15 and $700 \mathrm{~m}$. The quasar J0334-4008 was observed as a flux, bandpass, and pointing calibrator, while the quasar J03423007 served as phase calibrator. The observations were performed in 5 different frequency settings, covering the frequency range 84-115 GHz. This enables the observation of one or more CO lines over a wide range of redshifts (see Figure 2). Lower and upper side bands of the frequency settings partially overlap in the central part of the frequency range $(96-103 \mathrm{GHz})$, thus yielding improved sensitivity at these frequencies (see also Figure 3 in GL19).

\subsection{Data Reduction, Calibration, and Imaging}

We processed the data using both the CASA pipeline for ALMA data (v. 4.7.0; McMullin et al. 2007) and our own procedures (see, e.g., Aravena et al. 2016a), which follow the general scheme of the official ALMA pipeline. Our independent inspection for data to be flagged allowed us to improve the depth of our scan in one of the frequency settings by up to $20 \%$. In all the other frequency settings, the final rms appears consistent with the one computed from the cube provided by the ALMA pipeline. As the cube created with our own procedures is at least as good (in terms of low noise) as the one from the pipeline, we will refer to the former in the remainder of the analysis.

We imaged the $3 \mathrm{~mm}$ cube with natural weighting using the task tclean. The resulting synthesized beam is $\approx 1$ !" $75 \times 1$ ". 49

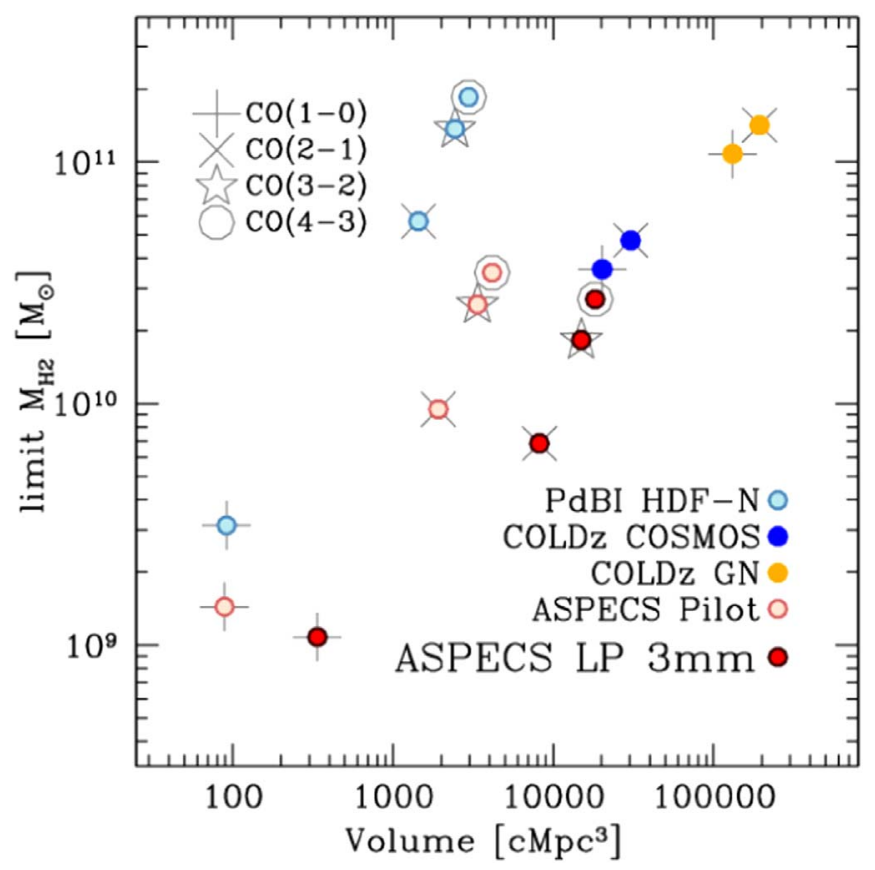

Figure 3. Depth and volume coverage of the molecular scans performed so far: the PdBI scan (Decarli et al. 2014; Walter et al. 2014), the COLDz survey (Pavesi et al. 2018; Riechers et al. 2019), the ASPECS Pilot (Decarli et al. 2016a; Walter et al. 2016), and the ASPECS LP $3 \mathrm{~mm}$ (this work). The $\mathrm{H}_{2}$ mass limits are computed at $5 \sigma$ in the case of line widths of $200 \mathrm{~km} \mathrm{~s}^{-1}$, assuming the CO SLED by Daddi et al. (2015) and a CO-to- $\mathrm{H}_{2}$ conversion factor $\alpha_{\mathrm{CO}}=3.6 M_{\odot}\left(\mathrm{K} \mathrm{km} \mathrm{s}^{-1} \mathrm{pc}^{2}\right)^{-1}$. Limits from various $\mathrm{CO}$ transitions are plotted. The complementarity of field coverage and depth in these campaigns is apparent.

$(\mathrm{PA}=91.5)$ at the center of the observed frequency range. The lack of very bright sources in our cubes allows us to perform our analysis on the "dirty" cube, thus preserving the intrinsic properties of the noise. The resulting cube is used for the line search, and in most of the following analysis. In addition, we image the data set after applying a $\mathrm{u}, \mathrm{v}$ taper of $3^{\prime \prime}$ (using the uvtaper parameter in tclean) cleaned to $2 \sigma$. This yields a reconstructed beam of $\sim 3$ " 5 . This latter cube is used only to extract the spectra of the sources identified in the search: thanks to the lower angular resolution, the spectra extracted in this way encapsulate all the emission of the sources, even in the case of sources that are spatially resolved in the naturally weighted imaging (see Aravena et al. 2019 for a discussion on the size of the CO emission in ASPECS LP $3 \mathrm{~mm}$ ).

We rebin the frequency dimension in channels of $7.813 \mathrm{MHz}$, i.e., $2 \times$ the native spectral resolution of the observations. At 99.5 GHz, this corresponds to $\Delta v \approx 23.5 \mathrm{~km} \mathrm{~s}^{-1}$. We use the "nearest" interpolation scheme in order to maintain the independence of the channels despite the small frequency corrections due to the Earth rotation and revolution during the execution of the observations. We reach a sensitivity of $\sim 0.2 \mathrm{mJy}_{\text {beam }}{ }^{-1}$ per $7.813 \mathrm{MHz}$ channel throughout the scanned frequency range. For a line width of $200 \mathrm{~km} \mathrm{~s}^{-1}$, these limits correspond to fiducial $5 \sigma$ $\mathrm{CO}$ line luminosity limits of $(1.4,2.1,2.3) \times 10^{9} \mathrm{~K} \mathrm{~km} \mathrm{~s}^{-1} \mathrm{pc}^{2}$, for $\mathrm{CO}(2-1), \mathrm{CO}(3-2)$, and $\mathrm{CO}(4-3)$, respectively. Via the working assumptions discussed in Section 4.3, we infer $\mathrm{H}_{2}$ mass limits of $6.8 \times 10^{9} M_{\odot}, 1.8 \times 10^{10} M_{\odot}$, and $2.7 \times 10^{10} M_{\odot}$ at $1.006<z<1.738,2.008<z<3.107$, and $3.011<z<4.475$ respectively. Figure 3 compares these molecular gas mass limits and volume coverage reached in ASPECS LP $3 \mathrm{~mm}$ with those of 
Table 1

CO Transitions, Redshift Bins, Cosmic Volume, and Typical $\mathrm{H}_{2}$ Mass Limit (at $5 \sigma$, Assuming a Line Width of $200 \mathrm{~km} \mathrm{~s}^{-1}$, CO Excitation as in Daddi et al. 2015, and a CO-to- $\mathrm{H}_{2}$ Conversion Factor $\left.\alpha_{\mathrm{CO}}=3.6 M_{\odot}\left(\mathrm{K} \mathrm{km} \mathrm{s}^{-1} \mathrm{pc}^{2}\right)^{-1}\right)$ in ASPECS LP $3 \mathrm{~mm}$

\begin{tabular}{lccc}
\hline \hline Line & Redshift & $\begin{array}{c}\text { Volume } \\
\left(\mathrm{cMpc}^{3}\right) \\
(3)\end{array}$ & $\begin{array}{c}\text { Limit } M_{\mathrm{H} 2} \\
\left(10^{10} M_{\odot}\right) \\
(4)\end{array}$ \\
\hline $\mathrm{CO}(1-0)$ & $(2)$ & 338 & 0.11 \\
$\mathrm{CO}(2-1)$ & $0.003-0.369$ & 8198 & 0.68 \\
$\mathrm{CO}(3-2)$ & $1.006-1.738$ & 14931 & 1.8 \\
$\mathrm{CO}(4-3)$ & $2.008-3.107$ & 18242 & 2.7 \\
\hline
\end{tabular}

all the other molecular scans performed so far. Table 1 lists the $\mathrm{CO}$ redshift coverage, fiducial gas mass limits, and the volume of universe of ASPECS LP $3 \mathrm{~mm}$ in various CO transitions.

\section{Ancillary Data}

The HUDF is one of the best studied extragalactic regions in the sky. Our observations thus benefit from a wealth of ancillary data of unparalleled quality in terms of depth, angular resolution, wavelength coverage, and richness of spectroscopic information. When comparing with literature multiwavelength catalogs, we apply a rigid astrometry offset $(\Delta$ R.A. $=+0$ " $076, \Delta$ decl. $=$ -0".279; see Rujopakarn et al. 2016; Dunlop et al. 2017) to available optical/NIR catalogs, in order to account for the different astrometric solution between the ALMA data and optical/NIR data.

The bulk of optical and NIR photometry comes from the Hubble Space Telescope (HST) Cosmic Assembly Nearinfrared Deep Extragalactic Legacy Survey (CANDELS; Grogin et al. 2011; Koekemoer et al. 2011). These are based both on archival and new HST images obtained with the Advanced Camera for Surveys (ACS) at optical wavelengths, and with the Wide Field Camera 3 (WFC3) in the near-infrared. We refer to the photometric compilation by Skelton et al. (2014), which also includes ground-based optical and NIR photometry from Nonino et al. (2009), Hildebrandt et al. (2006), Erben et al. (2005), Cardamone et al. (2010), Wuyts et al. (2008), Retzlaff et al. (2010), and Hsieh et al. (2012), as well as Spitzer IRAC $3.6 \mu \mathrm{m}, 4.5 \mu \mathrm{m}, 5.8 \mu \mathrm{m}$, and $8.0 \mu \mathrm{m}$ photometry from Dickinson et al. (2003), Elbaz et al. (2011), and Ashby et al. (2013). We also include the Spitzer MIPS $24 \mu \mathrm{m}$ photometric information from Whitaker et al. (2014).

The main optical spectroscopy sample in the ASPECS LP footprint comes from the MUSE Hubble Ultra Deep Survey (Bacon et al. 2017), a mosaic of nine contiguous fields observed with the Multi Unit Spectroscopic Explorer at the ESO Very Large Telescope. The surveyed area encompasses the entire HUDF. MUSE provides integral field spectroscopy of a $1^{\prime} \times 1^{\prime}$ square field over the wavelength range of 4750-9300 A. This yields emission-line redshift coverage in the ranges $z<0.857,0.274<z<1.495,1.488<z<3.872$, and $2.906<z<6.648$ for $[\mathrm{OIII}]_{5000 \AA}$, [OII $\left.]_{3727 \AA}, \mathrm{CIII}\right]_{1909 \AA}$, and $\operatorname{Ly} \alpha$, respectively. The redshift catalog based on the MUSE Hubble Ultra Deep Survey consists of $>1500$ galaxies with spectroscopic redshifts in the HUDF (Inami et al. 2017). We also include any additional spectroscopic information based on various studies at optical and NIR wavelengths, as compiled in Le Fèvre et al. (2005), Coe et al. (2006), Skelton et al. (2014), and Morris et al. (2015).
HST grism spectroscopy is also available in the HUDF. These observations allow for integral field spectroscopy with subarcsec angular resolution at relatively modest $(\lambda / \Delta \lambda \lesssim$ 1000) spectral resolution. While optical grism spectroscopy of the HUDF has been done (Xu et al. 2007), we take particular advantage of the more recent HST grism spectroscopy campaigns at NIR wavelengths, in particular, the 3D-HST survey (Momcheva et al. 2016). This complements the MUSE information, providing spectroscopy of $\mathrm{H} \alpha, \mathrm{H} \beta$, and other restframe optical lines at $z=1-3$, together with some additional redshift information in the "redshift desert" at $1.5<z<2.9$ where MUSE is less efficient due to the paucity of bright emission lines that are shifted into the MUSE wavelength range at these redshifts.

We create a master catalog of galaxies in the HUDF by combining the Skelton et al. (2014) catalog with the compilations by Le Fèvre et al. (2005), Coe et al. (2006), Xu et al. (2007), Rhoads et al. (2009), McLure et al. (2013), Schenker et al. (2013), Bouwens et al. (2014, 2015), Morris et al. (2015), and Inami et al. (2017). The catalogs are merged with a simple geometrical association, with an angular threshold of 0 ". 5 (1"! 0$)$ for the photometry (spectroscopy). This selection is also cross-matched with the measurements of morphological parameters (size, ellipticity, and light concentration index) from van der Wel et al. (2012). The whole catalog, extending over most of the GOODS-South footprint, consists of $>63,000$ entries. In the $2 ! 5 \times 2 ! .1$ area of the $\mathrm{XDF}$, the catalog includes photometry in $>30$ broad and medium bands for $\sim 7000$ galaxies, 475 of which have a spectroscopic redshift.

The photometric data set is modeled with the high- $z$ extension of the Spectral Energy Distribution (SED) fitting code MAGPHYS (da Cunha et al. 2008, 2015), in order to infer physical parameters: stellar mass, sSFR (and thus, SFR), dust extinction, IR luminosity, etc. We use the available photometry between $0.37 \mu \mathrm{m}$ and $8.0 \mu \mathrm{m}$, as well as data from the available $1.2 \mathrm{~mm}$ imaging of the field. These results are discussed in detail in Boogaard et al. (2019).

\section{Analysis and Results}

Our goal is to compute CO LFs and measurements of $\rho\left(\mathrm{H}_{2}\right)$ based on the results from the CO line search in the ASPECS LP $3 \mathrm{~mm}$ data. Our workflow, sketched in Figure 4, is articulated in four main blocks: the search for line candidates in the cube, and their characterization in terms of observed quantities (e.g., line fluxes); the assessment of the reliability of the line candidates and of the completeness of our line search; the identification of the line candidates and the measurement of a CO-based redshift; and the construction of high-level data products (e.g., LFs).

\subsection{Line Search}

We extensively discuss the line search approach in GL19, and summarize the main steps here for completeness. The cube is searched for emission at any spatial position and spectral coordinate, without any prior based on data from other wavelengths, in order to minimize biases in our selection function. Among the compilations presented in GL19, here we refer to the results obtained with findclumps. This catalog of line candidates consists of 613 entries at $\mathrm{S} / \mathrm{N}>5.0,70$ at $\mathrm{S} / \mathrm{N}>5.5,21$ at $\mathrm{S} / \mathrm{N}>6.0$, and 15 at $\mathrm{S} / \mathrm{N}>6.5$.

The fidelity or reliability of a line candidate gauges the impact of false positive detections in our search. The idea is to 


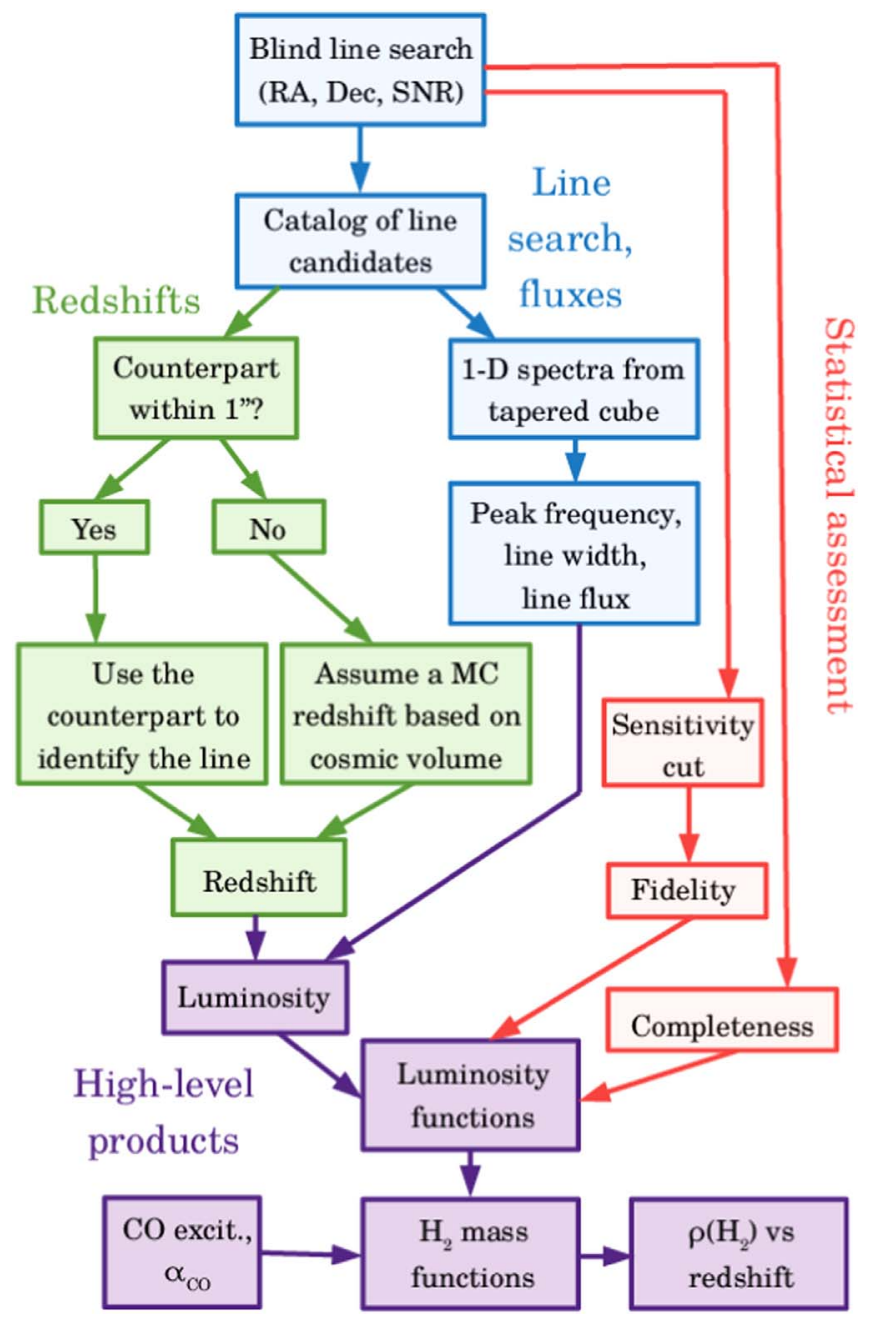

Figure 4. Scheme of the workflow followed in this analysis. Four broad areas are identified: the search of line candidates and their characterization in terms of observed quantities (in particular, the line flux), marked in blue; the redshift association, in green; the statistical analysis required to gauge the impact of false positives and of the incompleteness of our search, colored in red; and finally, the high-level data products, in purple.

estimate the probability that a given line candidate may be spurious (i.e., a noise feature). The statistics of negative line candidates is used to model the noise properties of the cube, as a function of the $\mathrm{S} / \mathrm{N}$ and the width of each line candidate. ${ }^{32}$ The fidelity is then defined as $1-P$, where $P$ is the probability of a (positive) line candidate to be due to noise. We limit our analysis to line candidates with fidelity $>20 \%$. We discuss the impact of fidelity on our results in Section 5.

The completeness of our line search is estimated by ingesting in the cube mock lines spanning a range of values for various parameters (3D position in the cube, flux, width), under the assumption that the lines are well described by Gaussian profiles. The line search is then repeated, and the completeness is simply inferred as the ratio between the number of retrieved and ingested mock lines, as a function of all the input parameters. In the construction of the CO LFs, we only consider line candidates with a parameter set yielding a completeness $>20 \%$.

\footnotetext{
${ }^{32}$ Since we adopt a matched-filter approach in the line search, the line width affects the reliability of the line candidates in that the narrower the filter kernel, the more independent resolution elements are present in the cube. As a consequence, the probability of finding a high-S/N noise peak increases.
}

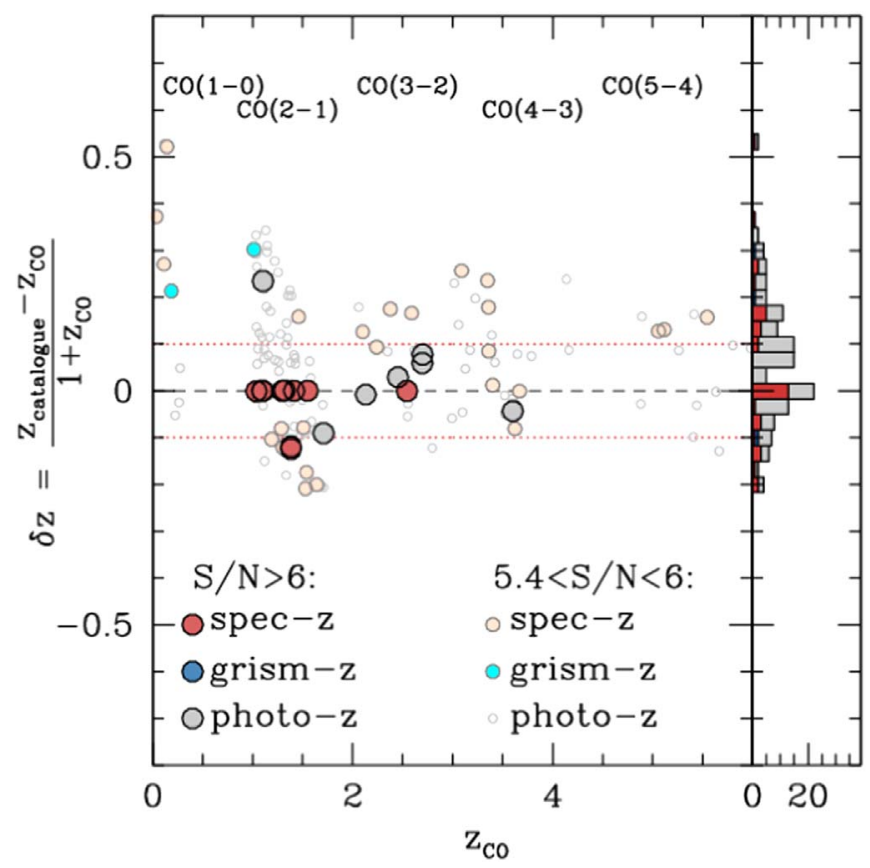

Figure 5. Comparison between the CO-based redshifts of the line candidates in our search, and the redshifts available in existing galaxy catalogs in the field. By construction, only line candidates with tentative counterparts are shown (141 line candidates). The panel on the right shows the collapsed distribution in $\delta z=\left(z_{\mathrm{cat}}-z_{\mathrm{CO}}\right) /\left(1+z_{\mathrm{CO}}\right)$. More than half of the sources $(79 / 141)$ lie within $|\delta z|<0.1$ (dotted, dark red lines). The largest deviations observed in spectroscopically confirmed redshifts are due to blends of overlapping galaxies along the line of sight (see Boogaard et al. 2019).

\subsection{Line Identification and Redshifts}

In order to convert the fluxes of the line candidates into luminosities, we need to identify the observed lines. In principle, the spectral range covered in our $3 \mathrm{~mm}$ scan is broad enough to encompass multiple $\mathrm{CO}$ transitions at specific redshifts, thus offering a robust direct constraint on the line identification. However, as shown in Figure 2, this happens only at relatively high redshifts $(z \gtrsim 3$, if one considers both $\mathrm{CO}$ and [CI]). We therefore need to consider different approaches to pin down the redshift of our line candidates. First, we search for a counterpart at optical/NIR wavelengths. If successful, we use the available redshift of the counterpart to associate line candidates and $\mathrm{CO}$ transitions: if the counterpart has a redshift $z_{\text {cat }}<0.8,0.8<$ $z_{\text {cat }}<1.9,1.9<z<3.2$, etc., we identify the line candidate as $\mathrm{CO}(1-0), \mathrm{CO}(2-1), \mathrm{CO}(3-2)$, etc., respectively. The majority of the 21 line candidates with $\mathrm{S} / \mathrm{N}>6$ show very good agreement $\left.|\delta z|=\left|\left(z_{\mathrm{cat}}-z_{\mathrm{CO}}\right) /\left(1+z_{\mathrm{CO}}\right)\right| \lesssim 0.01\right)$ between CO-based and catalog redshifts (see Figure 5). Other line candidates have a CO redshift roughly consistent $(|\delta z|<0.3)$ with the catalog photometric redshifts. Two galaxies detected at $\mathrm{S} / \mathrm{N}>6$ in $\mathrm{CO}$ have a spectroscopic catalog redshift that is inconsistent with the CO-based redshift. Our detailed analysis of the MUSE data confirms that these cases are examples of overlapping galaxies at different redshifts; i.e., both the catalog values and the CO-based values are confirmed (Boogaard et al. 2019). Figure 5 shows the comparison between the CO-based and catalog redshifts.

If the line candidates do not have counterparts at other wavelengths (about $25 \%$ of line candidates at $\mathrm{S} / \mathrm{N}>5$ ), the line identification is performed through a bootstrap, where the probability of a line candidate to be $\mathrm{CO}(1-0), \mathrm{CO}(2-1), \mathrm{CO}(3$ $-2)$, and $\mathrm{CO}(4-3)$ is proportional to the volume of universe 
sampled in each of these transitions with ASPECS LP at $3 \mathrm{~mm}$. We do not consider transitions at higher $J$ values, because significant $\mathrm{CO}$ excitation would have to be invoked in order to explain bright high- $J$ line emission. In Appendix B, we discuss the impact of these assumptions on our results.

In the construction of CO LFs, we only use CO-based redshifts.

\subsection{Line Luminosities and Corresponding $\mathrm{H}_{2}$ Mass}

The line fluxes are transformed into luminosities following Carilli \& Walter (2013):

$$
\begin{aligned}
\frac{L^{\prime}}{\mathrm{K} \mathrm{km} \mathrm{s}^{-1} \mathrm{pc}^{2}}= & \frac{3.257 \times 10^{7}}{1+z} \frac{F_{\text {line }}}{\mathrm{Jy} \mathrm{km} \mathrm{s}^{-1}}\left(\frac{\nu_{0}}{\mathrm{GHz}}\right)^{-2} \\
& \times\left(\frac{D_{\mathrm{L}}}{\mathrm{Mpc}}\right)^{2},
\end{aligned}
$$

where $F_{\text {line }}$ is the integrated line flux, $\nu_{0}$ is the rest-frame frequency of the line, and $D_{\mathrm{L}}$ is the luminosity distance. We then infer the corresponding $\mathrm{CO}(1-0)$ luminosities by adopting the $\mathrm{CO}[J-(J-1)]$-to-CO $(1-0)$ luminosity ratios, $r_{J 1}$, from Daddi et al. (2015): $L^{\prime}[\mathrm{CO}(1-0)]=L^{\prime} / r_{J 1}$, with $r_{J 1}=\{1.00,0.76 \pm$ $0.09,0.42 \pm 0.07,0.31 \pm 0.07\}$, for $J_{\text {up }}=\{1,2,3,4\}$. These values are based on VLA and PdBI observations of multiple CO transitions in four main-sequence galaxies at $z \approx 1.5$. These galaxies are less extreme than the typical, high IR luminosity galaxies studied in multiple $\mathrm{CO}$ transitions at $z>1$, and thus likely more representative of the galaxies studied here. We include a bootstrapped realization of the uncertainties on $r_{J 1}$ in the conversion. In Appendix B we discuss the impact of the $r_{J 1}$ assumptions on our results.

The cosmic microwave background at high redshift enhances the minimum temperature of the cold ISM, and suppresses the observability of $\mathrm{CO}$ lines in galaxies because of the lower contrast against the background (for extended discussions, see, e.g., da Cunha et al. 2013; Tunnard \& Greve 2016). The net effect is that the observed $\mathrm{CO}$ emission is only a fraction of the intrinsic one, with the suppression being larger for lower $\mathbf{J}$ transitions and at higher redshifts. This correction is, however, typically small at $z=1-3$, and often neglected in the literature (e.g., Tacconi et al. 2018). Indeed, for $T_{\text {kin }} \approx T_{\text {dust }}$, and following the $T_{\text {dust }}$ evolution in Magnelli et al. (2014), we find $T_{\text {kin }}>30 \mathrm{~K}$ at $z>1$, thus yielding CO flux corrections of $\lesssim 15 \%$ up to $z=4.5$. Because of its minimal impact, the associated uncertainties, and for consistency with the literature, we do not correct our measurements for the cosmic microwave background impact.

The resulting $\mathrm{CO}(1-0)$ luminosities are converted into molecular gas masses, $M_{\mathrm{H} 2}$, via the assumption of a CO-to- $\mathrm{H}_{2}$ conversion factor, $\alpha_{\mathrm{CO}}$ :

$$
M_{\mathrm{H} 2}=\frac{\alpha_{\mathrm{CO}}}{r_{J 1}} L^{\prime} .
$$

A widespread assumption in the literature on "normal" highredshift galaxies (e.g., Daddi et al. 2010a; Magnelli et al. 2012; Carilli \& Walter 2013; Tacconi et al. 2013, 2018; Genzel et al. 2015; Riechers et al. 2019) is a value of $\alpha_{\mathrm{CO}} \approx 4 M_{\odot}$ $\left(\mathrm{K} \mathrm{km} \mathrm{s}^{-1} \mathrm{pc}^{2}\right)^{-1}$, consistent with the Galactic value (see, e.g., Bolatto et al. 2013), once the helium contribution $(\sim 36 \%)$ is removed. Here we adopt $\alpha_{\mathrm{CO}}=3.6 M_{\odot}\left(\mathrm{K} \mathrm{km} \mathrm{s}^{-1} \mathrm{pc}^{2}\right)^{-1}$ (Daddi et al. 2010a). A different, yet constant choice of $\alpha_{\mathrm{CO}}$ would result in a linear scaling of our results involving $M_{\mathrm{H} 2}$ and $\rho\left(\mathrm{H}_{2}\right)$. This is further discussed in Section 5.

\subsection{CO LFs}

The CO LFs are constructed in a similar way as in Decarli et al. (2016a) via a Monte Carlo approach that allows us to simultaneously account for all the uncertainties in the line flux estimates, in the line identification, and in the conversion factors, as well as for the fidelity of the line candidates. For each line candidate, we compute the corresponding values of completeness and fidelity, based on the observed line properties $(\mathrm{S} / \mathrm{N}$, line width, flux, etc.). If the line has been confirmed by, e.g., a counterpart with a matching spectroscopic redshift, we assume that the fidelity is 1 . In all other cases, we conservatively treat our fidelity estimates as upper limits, and adopt a random value of fidelity that is uniformly distributed between 0 and such an upper limit (see GL19; Pavesi et al. 2018; Riechers et al. 2019). We extract a random number for each entry; line candidates are kept in our analysis only if the random value is below the fidelity threshold (thus, the lower the fidelity, the lower the chances that the line candidate is kept in our analysis). Typically, 20-40 line candidates survive this selection in each realization.

We split the list of line candidates by $\mathrm{CO}$ transitions and in 0.5 dex wide bins of luminosity. In each bin, we compute the Poissonian uncertainties. We then scale up each entry by the inverse of the completeness. The completeness-corrected entry counts in each bin are then divided by the comoving volume covered in each transition. This is computed by counting the area with sensitivity $>50 \%$ of the peak sensitivity obtained at the center of the mosaic in each channel.

The CO LFs are created 1000 times (both for the observed $\mathrm{CO}$ transitions, and for the corresponding $J=1 \rightarrow 0$ ground transition), each time with a different realization of all the parameters that are left uncertain (the fidelity and its error bars, the identification of lines without counterparts, the $r_{J 1}$ ratio, etc.). The analysis is then repeated five times after a shift of 0.1 dex of the luminosity bins, which allows us to remove the dependence of the reconstructed $\mathrm{CO}$ LFs from the bin definition. The final CO LFs are the averages of all the CO $\mathrm{LF}$ realizations. The $\mathrm{CO}$ and $\mathrm{CO}(1-0) \mathrm{LFs}$ are listed in Tables 3 and 4 and plotted in Figures 6 and 7.

The $\mathrm{H}_{2}$ mass functions in our analysis are simply obtained by scaling the $\mathrm{CO}(1-0) \mathrm{LFs}$ by the (fixed) $\alpha_{\mathrm{CO}}$ factor. We then sum the CO-based completeness-corrected $\mathrm{H}_{2}$ masses of each line candidate passing the fidelity threshold in bins of redshift, and we divide by the comoving volume in order to derive the cosmic gas molecular mass density, $\rho\left(\mathrm{H}_{2}\right)$. By construction, we do not extrapolate toward low $\mathrm{CO}$ luminosities/low $\mathrm{H}_{2}$ masses. However, in the following we will show that accounting for the faint end would only very marginally affect our results.

\subsection{Analytical Fits to the CO LFs}

We fit the observed CO LFs with a Schechter function (Schechter 1976), in the logarithmic form used in Riechers et al. (2019):

$$
\log \Phi\left(L^{\prime}\right)=\log \Phi_{*}+\alpha \log \left(\frac{L^{\prime}}{L_{*}^{\prime}}\right) \frac{1}{\ln 10} \frac{L^{\prime}}{L_{*}^{\prime}}+\log (\ln (10))
$$




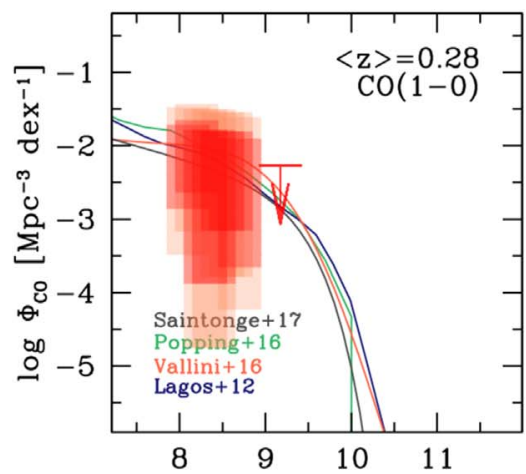

$\log \mathrm{L}_{\mathrm{co}}\left[\mathrm{K} \mathrm{km} \mathrm{s}^{-1} \mathrm{pc}^{2}\right]$

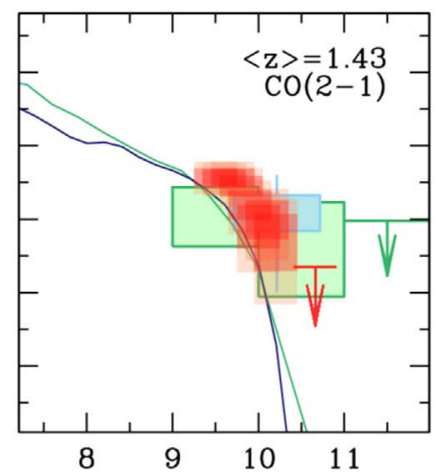

$\log \mathrm{L}_{\mathrm{co}}^{\prime}\left[\mathrm{K} \mathrm{km} \mathrm{s}^{-1} \mathrm{pc}^{2}\right]$

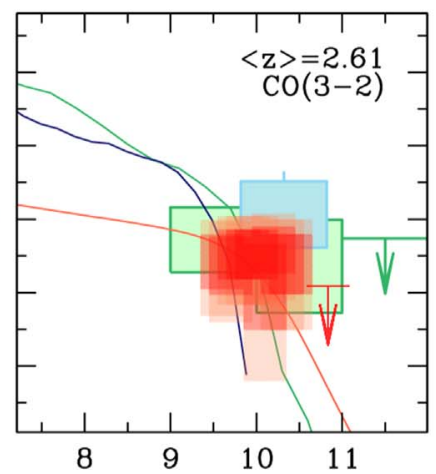

$\log \mathrm{L}_{\mathrm{co}}^{\prime}\left[\mathrm{K} \mathrm{km} \mathrm{s}^{-1} \mathrm{pc}^{2}\right]$

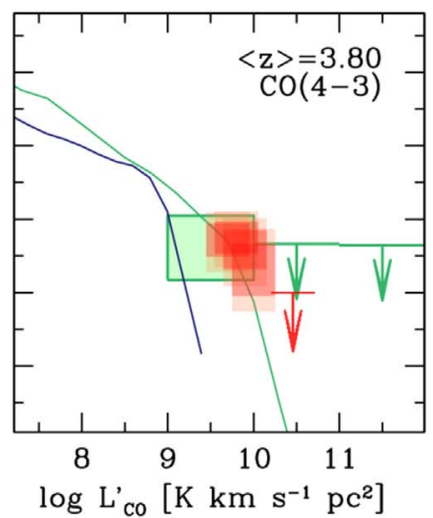

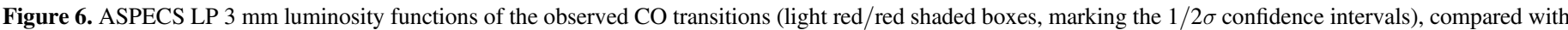

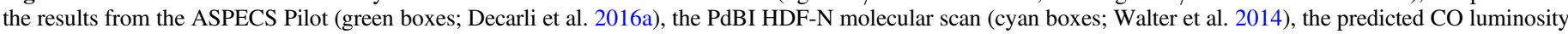

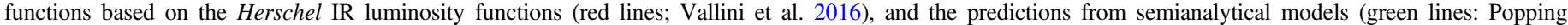

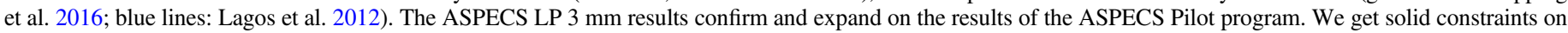

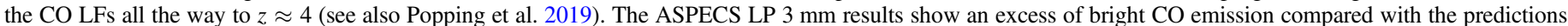
from models.
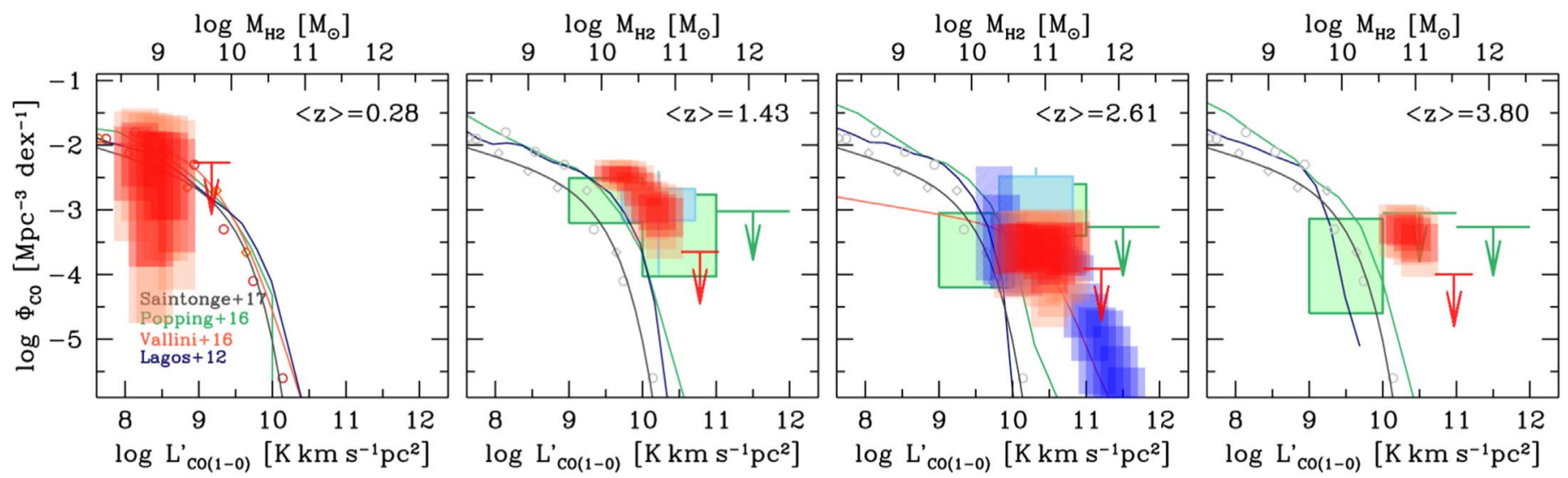

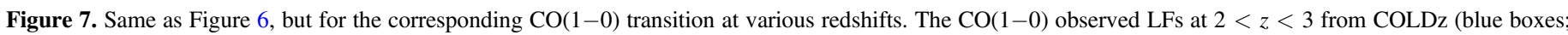

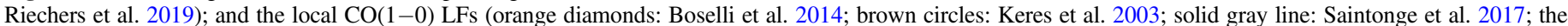

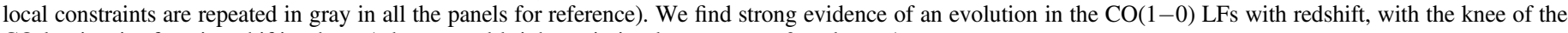
$\mathrm{CO}$ luminosity function shifting by $>1$ dex toward bright emission between $z \approx 0$ and $z>1$.

where $\Phi\left(L^{\prime}\right) d\left(\log L^{\prime}\right)$ is the number of galaxies per comoving volume with a CO line luminosity between $\log L^{\prime}$ and $\log L^{\prime}+d\left(\log L^{\prime}\right) ; \Phi_{*}$ is the scale number of galaxies per unit volume; $L_{*}^{\prime}$ is the scale line luminosity that sets the knee of the LF; and $\alpha$ is the slope of the faint end. We fit the observed CO LFs in the three redshift bins at $z>1$ considered in this study; the $z<0.37$ bin is ignored because of the modest luminosity range and sample size in our study. The LFs presented in this work are created in bins of 0.5 dex spaced by 0.1 dex, i.e., consecutive bins are not independent. In order to account for this, and to minimize the impact of our bin assumptions, we first fit the LFs using all the available bins, then we repeat the fits on the five independent contiguous subsets of the luminosity bins.

The slope of the faint end of the LF, $\alpha$, is very sensitive to the corrections we apply for fidelity and completeness (see the previous section). We therefore opt to conservatively use a fiducial fixed value of $\alpha=-0.2$ in our analysis. This is consistent with findings at $z \approx 0$ (Saintonge et al. 2017, once we take into account the different definition of $\alpha$ ), as well as with the typical slope of the stellar mass function of field galaxies at various redshifts (e.g.,
Ilbert et al. 2013). As for the other two parameters, we assume broad $(\sigma=0.5 \mathrm{dex}) \log$ normal distributions as priors in $\Phi_{*}$ and $L_{*}^{\prime}$, centered around $10^{-3} \mathrm{Mpc}^{-3} \mathrm{dex}^{-1}$, and $10^{9.5} \mathrm{~K} \mathrm{~km} \mathrm{~s}^{-1} \mathrm{pc}^{2}$, respectively. The best-fit value and the $1 \sigma$ confidence levels of the fitted parameters are derived from the 50\%, 14\%, and $86 \%$ quartiles of the marginalized posterior distributions of each parameter. They are listed in Table 2. Figure 8 compares the observed CO LFs with the fitted Schechter functions.

The fitted parameters do not show strong dependency on the choice of binning, with the results being typically consistent within $1 \sigma$ uncertainties. We find an indication of a higher $L_{*}^{\prime}$ at $z=1-3\left(\log L_{*}^{\prime}\left(\mathrm{K} \mathrm{km} \mathrm{s}^{-1} \mathrm{pc}^{2}\right) \approx 10.4\right)$ compared to the $z>3$ bin, and most importantly, with the local universe $\left(\log L_{*}^{\prime}\right.$ $\left(\mathrm{K} \mathrm{km} \mathrm{s}^{-1} \mathrm{pc}^{2}\right) \approx 9.9$, although with a different definition of the Schechter function; Saintonge et al. 2017).

The luminosity-weighted integral of the fitted LFs suggests that the ASPECS LP $3 \mathrm{~mm}$ data recover $83 \%, 91 \%$, and $71 \%$ of the total CO luminosity at $\langle z\rangle=1.43,2.61$, and at 3.80. In addition, if we adopt the best fit by Saintonge et al. (2017) for the lowest redshift bin, ASPECS LP $3 \mathrm{~mm}$ recovers 59\% of the total $\mathrm{CO}(1-0)$ luminosity in the local universe, although this 

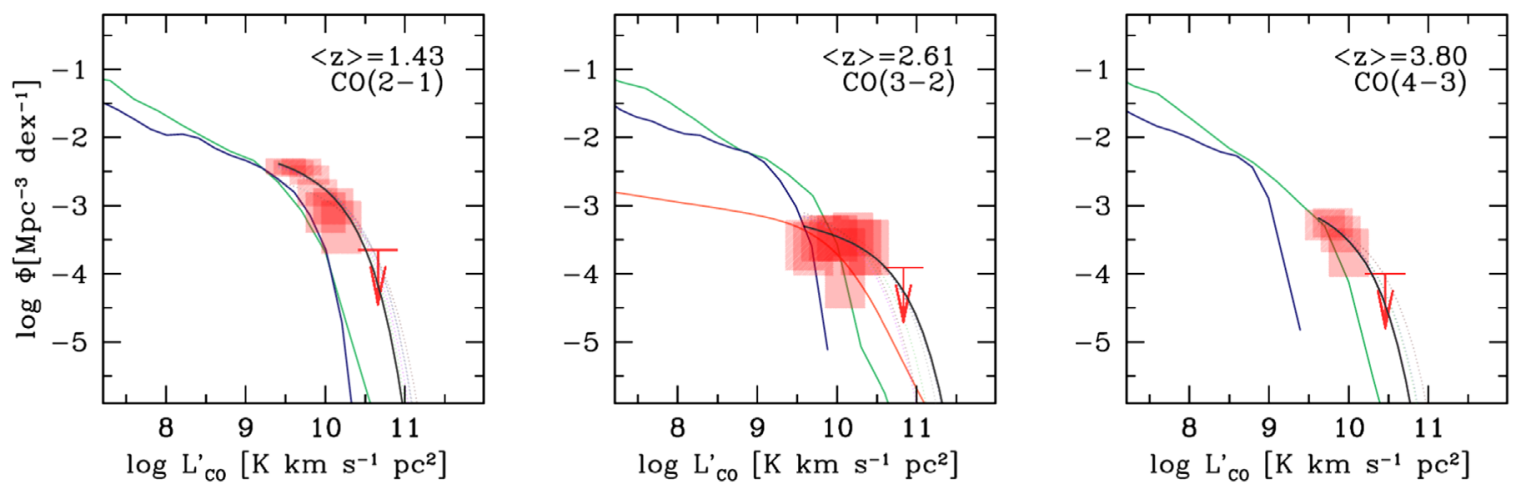

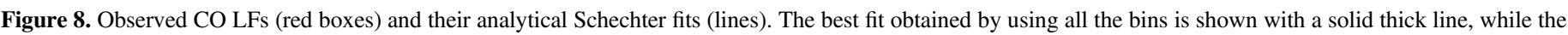

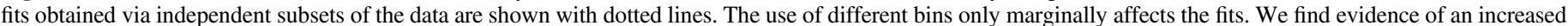

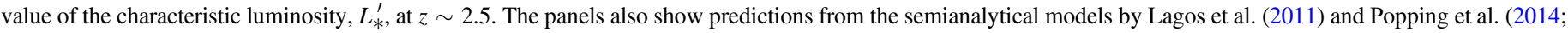
blue and green solid lines, respectively).

Table 2

Results of the Schechter Fits of the Observed CO LFs, Assuming a Fixed $\alpha=-0.2$

\begin{tabular}{|c|c|c|}
\hline Line & $\begin{array}{c}\log \Phi_{*} \\
\left(\mathrm{Mpc}^{-3} \mathrm{dex}^{-1}\right) \\
(2)\end{array}$ & $\begin{array}{c}\log L_{*}^{\prime} \\
\left(\mathrm{K} \mathrm{km} \mathrm{s}^{-1} \mathrm{pc}^{2}\right) \\
(3)\end{array}$ \\
\hline \multicolumn{3}{|c|}{ All $L^{\prime}$ bins } \\
\hline $\mathrm{CO}(2-1)$ & $-2.79_{-0.09}^{+0.09}$ & $10.09_{-0.09}^{+0.10}$ \\
\hline $\mathrm{CO}(3-2)$ & $-3.83_{-0.12}^{+0.13}$ & $10.60_{-0.15}^{+0.20}$ \\
\hline $\mathrm{CO}(4-3)$ & $-3.43_{-0.22}^{+0.19}$ & $9.98_{-0.14}^{+0.22}$ \\
\hline \multicolumn{3}{|c|}{ Independent $L^{\prime}$ bins } \\
\hline $\mathrm{CO}(2-1)$ & $-2.93_{-0.12}^{+0.11}$ & $10.23_{-0.11}^{+0.16}$ \\
\hline $\mathrm{CO}(2-1)$ & $-2.90_{-0.14}^{+0.16}$ & $10.22_{-0.22}^{+0.24}$ \\
\hline $\mathrm{CO}(2-1)$ & $-2.77_{-0.20}^{+0.21}$ & $10.12_{-0.25}^{+0.35}$ \\
\hline $\mathrm{CO}(2-1)$ & $-2.86_{-0.14}^{+0.15}$ & $10.17_{-0.17}^{+0.17}$ \\
\hline $\mathrm{CO}(2-1)$ & $-3.14_{-0.19}^{+0.19}$ & $10.32_{-0.18}^{+0.26}$ \\
\hline $\mathrm{CO}(3-2)$ & $-3.65_{-0.23}^{+0.25}$ & $10.49_{-0.22}^{+0.26}$ \\
\hline $\mathrm{CO}(3-2)$ & $-3.85_{-0.20}^{+0.21}$ & $10.59_{-0.20}^{+0.23}$ \\
\hline $\mathrm{CO}(3-2)$ & $-3.63_{-0.17}^{+0.17}$ & $10.36_{-0.21}^{+0.25}$ \\
\hline $\mathrm{CO}(3-2)$ & $-3.55_{-0.26}^{+0.28}$ & $10.22_{-0.21}^{+0.19}$ \\
\hline $\mathrm{CO}(3-2)$ & $-3.50_{-0.21}^{+0.22}$ & $10.24_{-0.15}^{+0.21}$ \\
\hline $\mathrm{CO}(4-3)$ & $-3.53_{-0.28}^{+0.36}$ & $10.01_{-0.21}^{+0.26}$ \\
\hline $\mathrm{CO}(4-3)$ & $-3.55_{-0.26}^{+0.23}$ & $10.10_{-0.16}^{+0.18}$ \\
\hline $\mathrm{CO}(4-3)$ & $-3.53_{-0.19}^{+0.18}$ & $10.08_{-0.15}^{+0.20}$ \\
\hline $\mathrm{CO}(4-3)$ & $-3.38_{-0.28}^{+0.26}$ & $9.98_{-0.20}^{+0.36}$ \\
\hline $\mathrm{CO}(4-3)$ & $-3.59_{-0.23}^{+0.25}$ & $10.21_{-0.25}^{+0.40}$ \\
\hline
\end{tabular}

last measure is strongly affected by cosmic variance due to the small volume probed by ASPECS LP $3 \mathrm{~mm}$.

\section{Discussion}

Figures 6 and 7 show that ASPECS LP $3 \mathrm{~mm}$ sampled a factor $\sim 20$ in CO luminosity at $z=1-4$ (see also Tables 3 and 4). We find evidence of an evolution in the CO LFs (and in the corresponding $\mathrm{CO}(1-0) \mathrm{LFs})$ as a function of redshift, compared to the local universe (Keres et al. 2003; Boselli et al. 2014; Saintonge et al. 2017), suggesting that the characteristic CO luminosity of galaxies at $z=1-4$ is an order of magnitude higher than in the local universe, once we account for $\mathrm{CO}$ excitation. This is in line with the findings from other studies, e.g., other molecular scans (Walter et al. 2014; Decarli et al. 2016a; Riechers et al. 2019); targeted CO observations on large samples of galaxies (e.g., Genzel et al. 2015; Aravena et al. 2016c; Tacconi et al. 2018); and similar works based on dust continuum observations (e.g., Gruppioni et al. 2013; Magnelli et al. 2013; Scoville et al. 2017). The CO LFs show an excess at the bright end compared with the predictions by semianalytical models (Lagos et al. 2011; Popping et al. 2014), and more compatible with empirical predictions (Sargent et al. 2014; Vallini et al. 2016). Figure 9 demonstrates that a prominent evolution in $\rho\left(\mathrm{H}_{2}\right)$ occurred between $z \approx 4$ and nowadays, with the molecular gas content in galaxies slowly rising since early cosmic epochs, peaking around $z=1-3$, and dropping by a factor of $6.5_{-1.4}^{+1.8}$ down to the present age (see also Table 5). The values of $\rho\left(\mathrm{H}_{2}\right)$ used here only refer to the actual line candidates, i.e., we do not attempt to extrapolate toward the undetected faint end of the LFs. However, as discussed in Section 4.5, our observations recover close to $90 \%$ of the total CO luminosity at $z=1.0-3.1$ (under the assumption of a slope of $\alpha=-0.2$ for the faint end), i.e., the derived $\rho\left(\mathrm{H}_{2}\right)$ values would shift upwards by small factors $(\sim 10 \%-20 \%)$. In Appendix B, we test the robustness of the CO LFs and $\rho\left(\mathrm{H}_{2}\right)$ evolution with redshift against some of the working assumptions in our analysis. A different choice of $\alpha_{\mathrm{CO}}$ would linearly affect our results on $\rho\left(\mathrm{H}_{2}\right)$. In particular, by adopting $\alpha_{\mathrm{CO}} \approx 2 M_{\odot}\left(\mathrm{K} \mathrm{km} \mathrm{s}^{-1} \mathrm{pc}^{2}\right)^{-1}$, as the comparison between dust-based and CO-based gas masses suggests (Aravena et al. 2019), we would infer a milder evolution of $\rho\left(\mathrm{H}_{2}\right)$ at $z>1$ and the local measurements.

In the following, we discuss our results in the context of previous studies.

\subsection{CO LFs}

Compared to any previous molecular scan at millimeter wavelengths (Walter et al. 2014; Decarli et al. 2016a; Riechers et al. 2019), ASPECS LP $3 \mathrm{~mm}$ provides superior sample statistics, which enables the more detailed analysis described in this series of papers. As shown in Figure 3, ASPECS LP $3 \mathrm{~mm}$ complements very well COLDz in that it samples a smaller volume but reaching a deeper sensitivity. The large volumes sampled by ASPECS LP $3 \mathrm{~mm}$ and COLDz, and the different targeted fields, mitigate the impact of cosmic variance. Overall, the CO LFs observed from the ASPECS LP $3 \mathrm{~mm}$ data appear in good agreement with the constraints from the first molecular scan observations (see Figure 6).

Figure 6 compares our observed CO LFs with the LF predictions by the semianalytical models presented in 


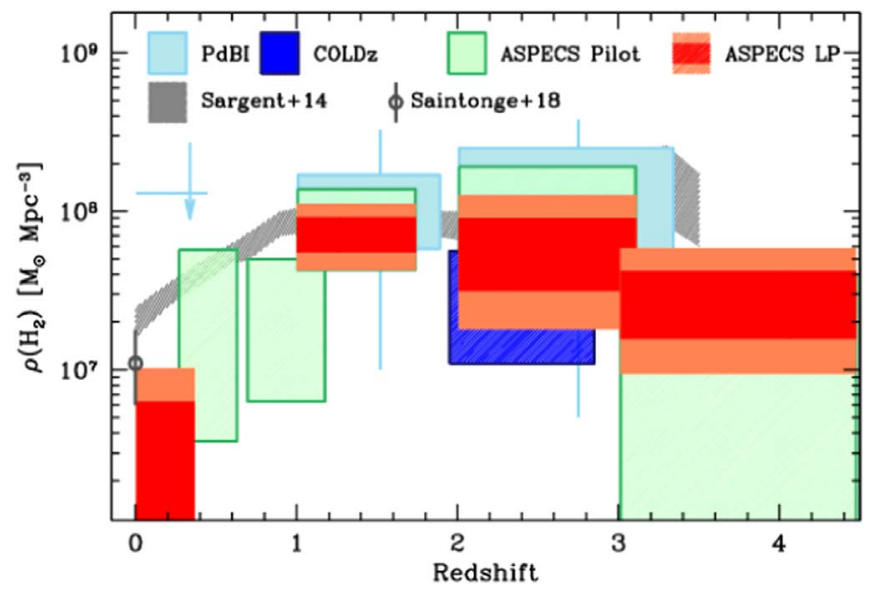

Figure 9. Redshift evolution of the cosmic molecular gas density, $\rho\left(\mathrm{H}_{2}\right)$, as constrained by ASPECS LP $3 \mathrm{~mm}$ (red shaded regions) and by other molecular scans: the PdBI scan (Walter et al. 2014), the COLDz survey (Riechers et al. 2019), and the ASPECS Pilot (Decarli et al. 2016a; shown in cyan, blue, and green boxes respectively), compared with the local measure by Saintonge et al. (2017; gray circle). The grading in the ASPECS LP boxes highlights the $1 \sigma$ and $2 \sigma$ confidence levels. The ASPECS LP $3 \mathrm{~mm}$ constraint on $\rho\left(\mathrm{H}_{2}\right)$ at $z<0.3$ is below the estimates from local studies, likely due to the small $L_{\mathrm{CO}}^{\prime}$ range sampled in ASPECS LP $3 \mathrm{~mm}$, and the higher impact of cosmic variance due to the small volume we probed. Our new data show that the molecular gas content slowly increases from early cosmic epochs up to $z \sim 1.5$, then dropped by a factor of $\sim 6$ to the present day. This is fully consistent with the constraints derived from other molecular scans, irrespective of the region of the sky they surveyed. The evolution appears more pronounced than what most semianalytical models predict (see, e.g., Lagos et al. 2011; Popping et al. 2014, and the discussion in Popping et al. 2019). The observed evolution in $\rho\left(\mathrm{H}_{2}\right)$ seems to closely match the evolution in $\rho_{\mathrm{SFR}}$ (Madau \& Dickinson 2014), thus suggesting that the gas content is the main driver of the star formation history.

Lagos et al. (2011) and Popping et al. (2014). Semianalytical models tend to underpredict the bright end of the CO LFs at $z>1$, with a larger discrepancy for the Lagos et al. (2011) models at $z>2$. The tension increases if we compare our inferred $\mathrm{CO}(1-0)$ LFs with the predictions from models (Figure 7). This hints at an intrinsic difference on how widespread large molecular gas reservoirs in high-redshift galaxies are as predicted by models, with respect to that suggested by our observations; the tension is somewhat reduced by the different treatment of the $\mathrm{CO}$ excitation (see Appendix B).

The $\mathrm{CO}(1-0) \mathrm{LF}$ inferred in our study at $2.0<z<3.1$ is in excellent agreement with the one derived from the COLDz survey (Riechers et al. 2019; see Figure 7). Because of the different parameter space, the COLDz data show larger uncertainties in the faint end, but provide a better constraint on the bright end compared to ASPECS LP $3 \mathrm{~mm}$. The good match between these two independent observations might be considered as supporting evidence that the impact of cosmic variance is relatively modest (the targeted fields are completely independent); and that our assumption on the $\mathrm{CO}$ excitation, used to transform $\mathrm{CO}(3-2)$ into its corresponding $\mathrm{CO}(1-0)$, works reasonably well. Interestingly, the $\mathrm{CO}(1-0) \mathrm{LFs}$ from ASPECS LP $3 \mathrm{~mm}$ and COLDz derived at $z \sim 2.5$ are in good agreement with the empirical predictions by Vallini et al. (2016), based on the Herschel IR LFs (Gruppioni et al. 2013).

It is also interesting to compare the inferred $\mathrm{CO}(1-0) \mathrm{LFs}$ with the ones measured in the local universe by Keres et al. (2003), Boselli et al. (2014), and Saintonge et al. (2017). The local measurements differ from each other by up to a factor of
2. Nevertheless, the ASPECS LP $3 \mathrm{~mm}$ data show a very clear evolution in the $\mathrm{CO}(1-0) \mathrm{LFs}$, with a shift upward of the knee of the LFs by an order of magnitude, or an excess by several orders of magnitude in the number density of bright $\left(L^{\prime}>10^{10} \mathrm{~K} \mathrm{~km} \mathrm{~s}^{-1} \mathrm{pc}^{2}\right) \mathrm{CO}(1-0)$ emitters at $z>1$ compared to the local universe.

\section{2. $\rho\left(\mathrm{H}_{2}\right)$ versus Redshift}

Figure 9 compares the observed evolution of $\rho\left(\mathrm{H}_{2}\right)$ as a function of redshift from the available molecular scan efforts. The ASPECS LP $3 \mathrm{~mm}$ data confirm the results from the PdBI scan in the HDF-N (Walter et al. 2014) and from the ASPECS Pilot (Decarli et al. 2016a), but with much tighter constraints thanks to the superior statistics. The cosmic density of molecular gas in galaxies appears to increase by a factor of $6.5_{-1.4}^{+1.8}$ from the local universe $\left[\rho\left(\mathrm{H}_{2}\right) \approx 1.1 \times 10^{7} M_{\odot} \mathrm{Mpc}^{-3}\right.$; Keres et al. 2003; Boselli et al. 2014; Saintonge et al. 2017] to $z \sim 1\left[\rho\left(\mathrm{H}_{2}\right) \approx\right.$ $\left.7.1 \times 10^{7} \mathrm{Mpc}^{-3}\right],{ }^{33}$ then follows a relatively flat evolution or possibly a mild decline toward higher redshifts. This is in excellent agreement with the constraints on $\rho\left(\mathrm{H}_{2}\right)$ from COLDz (Riechers et al. 2019) at $2.0<z<2.8$, and with the empirical predictions derived by Sargent et al. (2014) based on the "2-star formation mode" framework, where the distributions of various galaxy properties (gas fraction, star formation efficiency, metallicity, etc.) are inferred based on empirical relations, with a key role due to the offset of galaxies with respect to the "main sequence." This analysis results in a similar evolution of $\rho\left(\mathrm{H}_{2}\right)$ with redshift as the one found in ASPECS LP $3 \mathrm{~mm}$.

The observed evolution in $\rho\left(\mathrm{H}_{2}\right)$ is also in qualitative agreement with other observational studies. For example, most studies searching for $\mathrm{CO}$ emission in targeted observations of main-sequence galaxies find that $z=1-3$ galaxies typically have 5-10 times more gas than galaxies of similar stellar mass in the local universe (see, e.g., Genzel et al. 2015; Schinnerer et al. 2016; Tacconi et al. 2018). This is in line with the ASPECS LP $3 \mathrm{~mm}$ results, which point to a larger molecular gas content in typical galaxies at $z>1$ (see also Aravena et al. 2019). A similar trend is also reported by studies tackling the problem using dust as a probe of the gas content in high- $z$ galaxies (e.g., Gruppioni et al. 2013; Magnelli et al. 2013). For example, Scoville et al. (2017) put indirect constraints on $\rho\left(\mathrm{H}_{2}\right)$ at various redshifts using dust continuum measurements of Herschel-selected galaxies, and scaling by an internally calibrated dust-to-gas ratio. The evolution of $\rho\left(\mathrm{H}_{2}\right)$ that Scoville et al. (2017) infer is qualitatively similar, although somewhat shallower than the one observed in ASPECS LP $3 \mathrm{~mm}$, spanning only a factor of $\sim 2.5$ in $\rho\left(\mathrm{H}_{2}\right)$ compared to a factor $\sim 6.0$ found in ASPECS LP $3 \mathrm{~mm}$.

\section{Conclusions}

We presented the ASPECS LP $3 \mathrm{~mm}$ survey, an ALMA molecular scan encompassing most of the Hubble XDF over a large fraction of the $3 \mathrm{~mm}$ transparent band of the atmosphere. We exploited our data to search for massive molecular gas reservoirs (as traced by $\mathrm{CO}$ emission) in galaxies throughout $\sim 90 \%$ of cosmic history, with no prior on counterparts at other

\footnotetext{
33 The ASPECS LP $3 \mathrm{~mm}$ constraint on $\rho\left(\mathrm{H}_{2}\right)$ at $z<0.3$ is below the estimates from local studies; this is likely due to the fact that we only sampled a small luminosity range in $L_{\mathrm{CO}}^{\prime}$ in this redshift bin, in a tiny cosmic volume; furthermore, the HUDF was originally chosen to be relatively underdense of nearby galaxies.
} 
wavelengths. We detected 70 line candidates with $\mathrm{S} / \mathrm{N}>5.5$, $>75 \%$ of which have a photometric counterpart at optical/NIR wavelengths. This search allowed us to put stringent constraints on the CO LFs in various redshift bins, as well as to infer the cosmic density of molecular gas in galaxies, $\rho\left(\mathrm{H}_{2}\right)$. We found that:

(i) The CO LFs undergo significant evolution compared to the local universe. High redshift galaxies appear brighter in $\mathrm{CO}$ than galaxies in the local universe. In particular, at $z=1-3$, the characteristic $\mathrm{CO}(2-1)$ and $\mathrm{CO}(3-2)$ luminosity is $>3 \times$ higher than the characteristic $\mathrm{CO}(1$ $-0)$ luminosity observed in the local universe. The evolution is even stronger if we account for $\mathrm{CO}$ excitation. Analytical fits of our results suggest that we recovered the majority (up to $90 \%$, depending on assumptions on the faint end) of the total CO luminosity at $z=1.0-3.1$.

(ii) Similarly, $\rho\left(\mathrm{H}_{2}\right)$ shows a clear evolution with cosmic time: It slowly increased since early cosmic epochs, reached a peak around $z=1-3$, and then decreased by a factor of $6.5_{-1.4}^{+1.8}$ to the present day. This factor changes if $\alpha_{\mathrm{CO}}$ is allowed to evolve with redshift. In particular, the factor would be $\sim 3$ if we adopt $\alpha_{\mathrm{CO}}=2 M_{\odot}$ $\left(\mathrm{K} \mathrm{km} \mathrm{s}^{-1} \mathrm{pc}^{2}\right)^{-1}$ for galaxies at $z>1$.

(iii) Our results are in agreement with those of other molecular scans that targeted different regions of the sky and sampled different parts of the parameter space (in terms of depth, volume, transitions, etc.). Similarly, we generally confirm empirical predictions based on dust continuum observations and SED modeling.

(iv) Our results are in tension with predictions by semianalytical models, which struggle to reproduce the bright end of the observed CO LFs. The discrepancy might be mitigated with different assumptions on the $\mathrm{CO}$ excitation and $\alpha_{\mathrm{CO}}$. Popping et al. (2019) quantitatively address the comparison between models and the ASPECS LP $3 \mathrm{~mm}$ observations and the underlying assumptions of both.

(v) Our results hold valid if we restrict our analysis to the subset of galaxies with counterparts at redshifts that strictly match those inferred from our $\mathrm{CO}$ observations. The results are qualitatively robust against different assumptions concerning the $\mathrm{CO}$ excitation.

The observed evolution of $\rho\left(\mathrm{H}_{2}\right)$ is in quantitative agreement with the evolution of the cosmic star formation rate density ( $\rho_{\mathrm{SFR}}$; see, e.g., Madau \& Dickinson 2014), which also shows a mild increase up to $z=1-3$, followed by a drop by a factor of $\approx 8$ down to present day. Given that the star formation rate can be expressed as the product of the star formation efficiency (=star formation per unit gas mass) and the gas content mass, the similar evolution of $\rho\left(\mathrm{H}_{2}\right)$ and $\rho_{\mathrm{SFR}}$ leaves little room for a significant evolution of the star formation efficiency throughout $85 \%$ of cosmic history $(z \approx 3)$, at least when averaged over the entire galaxy population. The history of cosmic star formation appears dominated by the evolution in the molecular gas content of galaxies.

We thank the anonymous referee for useful feedback, which allowed us to improve the quality of the paper. The Geryon cluster at the Centro de Astro-Ingenieria UC was extensively used for the calculations performed in this paper. BASAL CATA PFB-06, the Anillo ACT-86, FONDEQUIP AIC-57, and QUIMAL 130008 provided funding for several improvements to the Geryon cluster. Este trabajo contó con el apoyo de CONICYT + Programa de Astronomía + Fondo CHINA-CONICYT. J.G.L. acknowledges partial support from ALMA-CONICYT project 31160033. D.R. acknowledges support from the National Science Foundation under grant No. AST-1614213. F.E.B. acknowledges support from CONICYT-Chile Basal AFB-170002 and the Ministry of Economy, Development, and Tourism's Millennium Science Initiative through grant IC120009, awarded to The Millennium Institute of Astrophysics, MAS. I.R.S. acknowledges support from the ERC Advanced Grant DUSTYGAL (321334) and STFC (ST/P000541/1). T.D.-S. acknowledges support from ALMACONICYT project 31130005 and FONDECYT project 1151239. J.H. acknowledges support of the VIDI research programme with project number 639.042.611, which is (partly) financed by the Netherlands Organisation for Scientific Research (NWO).

Facilities: ALMA data: 2016.1.00324.L. ALMA is a partnership of ESO (representing its member states), NSF (USA) and NINS (Japan), together with NRC (Canada), NSC and ASIAA (Taiwan), and KASI (Republic of Korea), in cooperation with the Republic of Chile. The Joint ALMA Observatory is operated by ESO, AUI/NRAO and NAOJ.

\section{Appendix A Measured CO LFs}

For the sake of reproducibility of our results, Table 3 reports the measured CO LFs in ASPECS LP $3 \mathrm{~mm}$. Similarly, Table 4 provides the inferred $\mathrm{CO}(1-0) \mathrm{LFs}$ from this study. Table 5 lists the estimated values of $\rho\left(\mathrm{H}_{2}\right)$ in various redshift bins and under different working hypotheses (see Appendix B). Finally, Table 6 lists the entries of the line candidates used in the construction of the LFs.

Table 3

Luminosity Functions of the Observed CO Transitions

\begin{tabular}{|c|c|c|c|c|c|}
\hline $\begin{array}{l}\log L^{\prime} \\
\left(\mathrm{K} \mathrm{km} \mathrm{s}^{-1} \mathrm{pc}^{2}\right) \\
\text { (1) }\end{array}$ & $\begin{array}{c}\log \Phi, 1 \sigma \\
\left(\operatorname{dex}^{-1} \mathrm{cMpc}^{-3}\right) \\
(2)\end{array}$ & $\begin{array}{c}\log \Phi, 2 \sigma \\
\left(\operatorname{dex}^{-1} \mathrm{cMpc}^{-3}\right) \\
(3)\end{array}$ & $\begin{array}{c}\log L^{\prime} \\
\left(\mathrm{K} \mathrm{km} \mathrm{s}^{-1} \mathrm{pc}^{2}\right) \\
\text { (4) }\end{array}$ & $\begin{array}{c}\log \Phi, 1 \sigma \\
\left(\operatorname{dex}^{-1} \mathrm{cMpc}^{-3}\right) \\
(5)\end{array}$ & $\begin{array}{c}\log \Phi, 2 \sigma \\
\left(\operatorname{dex}^{-1} \mathrm{cMpc}^{-3}\right) \\
(6)\end{array}$ \\
\hline & \multicolumn{2}{|c|}{$\mathrm{CO}(1-0)$} & & \multicolumn{2}{|c|}{$\mathrm{CO}(2-1)$} \\
\hline 8.1 & $-2.86-1.72$ & $-3.48-1.48$ & 9.5 & $-2.54-2.31$ & $-2.66-2.22$ \\
\hline 8.2 & $-2.64-1.65$ & $-3.17-1.43$ & 9.6 & $-2.58-2.33$ & $-2.69-2.24$ \\
\hline 8.3 & $-3.88-1.79$ & $-4.75-1.51$ & 9.7 & $-2.55-2.31$ & $-2.67-2.22$ \\
\hline 8.6 & $-3.15-1.81$ & $-3.78-1.55$ & 10.0 & $-3.40-2.81$ & $-3.72-2.64$ \\
\hline 8.7 & $-3.65-1.91$ & $-4.23-1.61$ & 10.1 & $-3.27-2.75$ & $-3.56-2.59$ \\
\hline 8.8 & $-3.65-1.91$ & $-4.23-1.61$ & 10.2 & $-3.70-2.93$ & $-4.16-2.73$ \\
\hline
\end{tabular}


Table 3

(Continued)

\begin{tabular}{|c|c|c|c|c|c|}
\hline $\begin{array}{l}\log L^{\prime} \\
\left(\mathrm{K} \mathrm{km} \mathrm{s}^{-1} \mathrm{pc}^{2}\right) \\
\text { (1) }\end{array}$ & $\begin{array}{c}\log \Phi, 1 \sigma \\
\left(\operatorname{dex}^{-1} \mathrm{cMpc}^{-3}\right) \\
(2)\end{array}$ & $\begin{array}{c}\log \Phi, 2 \sigma \\
\left(\operatorname{dex}^{-1} \mathrm{cMpc}^{-3}\right) \\
(3)\end{array}$ & $\begin{array}{c}\log L^{\prime} \\
\left(\mathrm{K} \mathrm{km} \mathrm{s}^{-1} \mathrm{pc}^{2}\right) \\
(4)\end{array}$ & $\begin{array}{c}\log \Phi, 1 \sigma \\
\left(\operatorname{dex}^{-1} \mathrm{cMpc}^{-3}\right) \\
(5)\end{array}$ & $\begin{array}{c}\log \Phi, 2 \sigma \\
\left(\operatorname{dex}^{-1} \mathrm{cMpc}^{-3}\right) \\
(6)\end{array}$ \\
\hline $\begin{array}{l}8.9 \\
9.0\end{array}$ & $\begin{array}{l}-3.65-1.91 \\
-5.52-1.97\end{array}$ & $\begin{array}{l}-4.23-1.61 \\
-6.39-1.65\end{array}$ & $\begin{array}{l}10.3 \\
10.4 \\
10.5 \\
\end{array}$ & $\begin{array}{l}-3.76-2.95 \\
-3.76-2.95 \\
-3.76-2.95 \\
\end{array}$ & $\begin{array}{l}-4.25-2.75 \\
-4.25-2.75 \\
-4.25-2.75 \\
\end{array}$ \\
\hline \multicolumn{4}{|c|}{$\mathrm{CO}(3-2)$} & \multicolumn{2}{|c|}{$\mathrm{CO}(4-3)$} \\
\hline 9.6 & $-3.95-3.21$ & $-4.31-3.01$ & 9.6 & $-3.56-3.09$ & $-3.78-2.93$ \\
\hline 9.7 & $-4.03-3.24$ & $-4.41-3.03$ & 9.7 & $-3.51-3.06$ & $-3.72-2.91$ \\
\hline 9.8 & $-3.82-3.14$ & $-4.16-2.96$ & 9.8 & $-3.46-3.04$ & $-3.65-2.89$ \\
\hline 9.9 & $-3.82-3.14$ & $-4.16-2.96$ & 9.9 & $-3.68-3.15$ & $-3.91-2.99$ \\
\hline 10.0 & $-3.82-3.14$ & $-4.16-2.96$ & 10.0 & $-4.04-3.34$ & $-4.26-3.13$ \\
\hline 10.1 & $-4.51-3.34$ & $-5.20-3.11$ & 10.1 & $-4.04-3.34$ & $-4.26-3.13$ \\
\hline 10.2 & $-3.74-3.10$ & $-4.10-2.92$ & 10.2 & $-4.17-3.40$ & $-4.35-3.18$ \\
\hline 10.3 & $-4.02-3.21$ & $-4.51-3.01$ & 10.3 & $-5.19-3.59$ & $-6.06-3.31$ \\
\hline 10.4 & $-4.02-3.21$ & $-4.51-3.01$ & & & \\
\hline
\end{tabular}

Note. $(1,5)$ Luminosity bin center; each bin is 0.5 dex wide. $(2-4,6-8)$ CO luminosity functions, reported as the minimum and maximum values of the confidence levels at $1 \sigma, 2 \sigma$, and $3 \sigma$.

Table 4

Inferred $\mathrm{CO}(1-0)$ Luminosity Functions in Various Redshift Bins

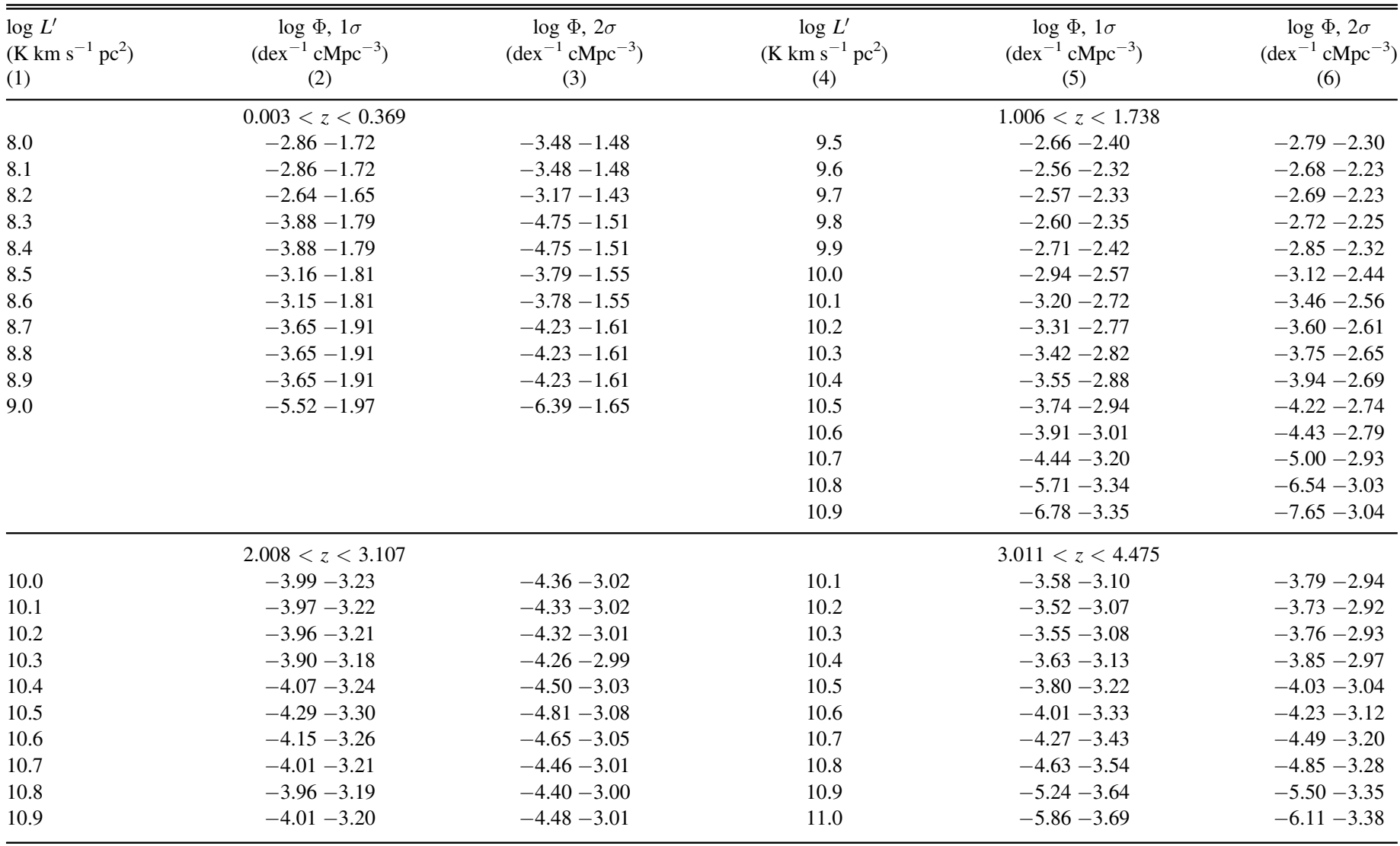

Note. $(1,4)$ Luminosity bin center; each bin is 0.5 dex wide. $(2-3,5-6)$ CO luminosity functions, reported as the minimum and maximum values of the confidence levels at $1 \sigma$ and $2 \sigma$. 
Table 5

Constraints on $\rho\left(\mathrm{H}_{2}\right)$ in Various Redshift Bins

\begin{tabular}{lcc}
\hline \hline $\begin{array}{l}\text { Redshift } \\
\text { bin }\end{array}$ & $\begin{array}{c}\log \rho\left(\mathrm{H}_{2}\right), 1 \sigma \\
\left(M_{\odot} \mathrm{Mpc}^{-3}\right) \\
(2)\end{array}$ & $\begin{array}{c}\log \rho\left(\mathrm{H}_{2}\right), 2 \sigma \\
\left(M_{\odot} \mathrm{Mpc}^{-3}\right) \\
(3)\end{array}$ \\
\hline & Reference estimate \\
$0.003-0.369$ & $5.89-6.80$ & $5.40-7.01$ \\
$1.006-1.738$ & $7.74-7.96$ & $7.63-8.05$ \\
$2.008-3.107$ & $7.50-7.96$ & $7.26-8.10$ \\
$3.011-4.475$ & $7.20-7.62$ & $6.97-7.77$ \\
\hline & Secure sources only & \\
$0.003-0.369$ & $5.13-6.41$ & $4.25-6.65$ \\
$1.006-1.738$ & $7.64-7.90$ & $7.51-7.99$ \\
$2.008-3.107$ & $7.39-7.96$ & $7.08-8.12$ \\
$3.011-4.475$ & $6.71-7.35$ & $6.37-7.53$ \\
\hline & Thermalized CO excitation & \\
$0.003-0.369$ & $5.89-6.80$ & $5.40-7.02$ \\
$1.006-1.738$ & $7.59-7.81$ & $7.47-7.90$ \\
$2.008-3.107$ & $7.03-7.48$ & $6.79-7.63$ \\
$3.011-4.475$ & $6.70-7.11$ & $6.48-7.25$ \\
\hline & Milky Way-like CO excitation & \\
$0.003-0.369$ & $5.91-6.82$ & $5.43-7.04$ \\
$1.006-1.738$ & $7.90-8.12$ & $7.79-8.20$ \\
$2.008-3.107$ & $7.65-8.09$ & $7.41-8.24$ \\
$3.011-4.475$ & $7.33-7.81$ & $7.08-7.96$ \\
\hline
\end{tabular}

Note. The quoted ranges correspond to the $1 \sigma$ and $2 \sigma$ confidence levels in our analysis. We provide our reference estimates based on the whole sample, and assuming intermediate CO excitation (Daddi et al. 2015; see Figure 9), as well as the estimates for the secure sources only (Figure 10) and for the whole sample, but using different assumptions for the $\mathrm{CO}$ excitation (Figure 11).

Table 6

Example of the Line Candidates Entering One of the Realizations of the CO LFs

\begin{tabular}{|c|c|c|c|c|c|c|c|c|}
\hline $\begin{array}{l}\text { R.A. } \\
\text { (deg) } \\
\text { (1) }\end{array}$ & $\begin{array}{c}\text { Decl. } \\
(\mathrm{deg}) \\
(2)\end{array}$ & $\begin{array}{l}z_{\mathrm{CO}} \\
(3)\end{array}$ & $\begin{array}{l}\mathrm{S} / \mathrm{N} \\
(4)\end{array}$ & $\begin{array}{l}\text { Compl. } \\
\text { (5) }\end{array}$ & $\begin{array}{l}\mathrm{c} / \mathrm{p} ? \\
(6)\end{array}$ & $\begin{array}{l}\text { fid. } \\
\text { (7) }\end{array}$ & $\begin{array}{c}L^{\prime} \\
\left(\mathrm{K} \mathrm{km} \mathrm{s}^{-1} \mathrm{pc}^{2}\right)\end{array}$ & $\begin{array}{l}J_{\text {up }} \\
\text { (9) }\end{array}$ \\
\hline 53.16063 & -27.77626 & 2.5436 & 36.18 & 1.00 & $\mathrm{Y}$ & 1.00 & $2.69 \times 10^{10}$ & $\overline{3}$ \\
\hline 53.17664 & -27.78551 & 1.3168 & 17.50 & 1.00 & $\mathrm{Y}$ & 1.00 & $8.77 \times 10^{9}$ & 2 \\
\hline 53.17086 & -27.77545 & 2.4534 & 15.25 & 1.00 & $\mathrm{Y}$ & 1.00 & $1.02 \times 10^{10}$ & 3 \\
\hline 53.14350 & -27.78324 & 1.4144 & 14.74 & 1.00 & $\mathrm{Y}$ & 1.00 & $1.78 \times 10^{10}$ & 2 \\
\hline 53.16569 & -27.76991 & 1.5502 & 13.98 & 1.00 & $\mathrm{Y}$ & 1.00 & $1.82 \times 10^{10}$ & 2 \\
\hline 53.16616 & -27.78754 & 1.0952 & 11.98 & 1.00 & $\mathrm{Y}$ & 1.00 & $6.17 \times 10^{9}$ & 2 \\
\hline 53.18138 & -27.77756 & 2.6956 & 9.95 & 1.00 & $\mathrm{Y}$ & 1.00 & $2.71 \times 10^{10}$ & 3 \\
\hline 53.14822 & -27.77389 & 1.3822 & 9.15 & 1.00 & $\mathrm{~N}$ & 1.00 & $3.17 \times 10^{9}$ & 2 \\
\hline 53.17908 & -27.78062 & 1.0365 & 8.74 & 1.00 & Y & 1.00 & $7.85 \times 10^{9}$ & 2 \\
\hline 53.15085 & -27.77440 & 1.3827 & 7.44 & 1.00 & $\mathrm{~N}$ & 1.00 & $3.64 \times 10^{9}$ & 2 \\
\hline 53.16583 & -27.78157 & 1.0964 & 7.31 & 1.00 & $\mathrm{Y}$ & 1.00 & $1.97 \times 10^{9}$ & 2 \\
\hline 53.14817 & -27.78451 & 3.6013 & 7.12 & 0.96 & $\mathrm{Y}$ & 1.00 & $5.59 \times 10^{9}$ & 4 \\
\hline 53.14523 & -27.77801 & 1.0985 & 7.10 & 0.85 & $\mathrm{Y}$ & 1.00 & $5.08 \times 10^{9}$ & 2 \\
\hline 53.15199 & -27.77552 & 1.0963 & 6.78 & 1.00 & $\mathrm{Y}$ & 1.00 & $3.56 \times 10^{9}$ & 2 \\
\hline 53.16635 & -27.76873 & 1.2942 & 6.11 & 1.00 & $\mathrm{Y}$ & 0.96 & $4.57 \times 10^{9}$ & 2 \\
\hline 53.17966 & -27.78428 & 0.1129 & 5.96 & 1.00 & $\mathrm{~N}$ & 0.95 & $1.04 \times 10^{8}$ & 1 \\
\hline 53.15192 & -27.78900 & 1.4953 & 5.91 & 1.00 & $\mathrm{Y}$ & 0.95 & $5.14 \times 10^{9}$ & 2 \\
\hline 53.14544 & -27.78721 & 1.1746 & 5.78 & 0.87 & $\mathrm{Y}$ & 0.93 & $3.08 \times 10^{9}$ & 2 \\
\hline 53.16513 & -27.76394 & 1.1769 & 5.77 & 1.00 & $\mathrm{~N}$ & 0.75 & $5.15 \times 10^{9}$ & 2 \\
\hline 53.15104 & -27.78691 & 1.7009 & 5.72 & 0.95 & Y & 0.87 & $4.04 \times 10^{9}$ & 2 \\
\hline 53.14553 & -27.77757 & 3.6038 & 5.66 & 0.92 & $\mathrm{Y}$ & 0.61 & $5.25 \times 10^{9}$ & 4 \\
\hline 53.15495 & -27.78709 & 1.0341 & 5.63 & 1.00 & $\mathrm{~N}$ & 0.36 & $3.39 \times 10^{9}$ & 2 \\
\hline 53.14659 & -27.77822 & 1.3821 & 5.56 & 0.93 & $\mathrm{~N}$ & 0.76 & $3.25 \times 10^{9}$ & 2 \\
\hline 53.15702 & -27.78166 & 1.1298 & 5.52 & 1.00 & $\mathrm{~N}$ & 0.72 & $2.56 \times 10^{9}$ & 2 \\
\hline 53.17554 & -27.78809 & 1.3835 & 5.46 & 0.46 & $\mathrm{~N}$ & 0.74 & $4.05 \times 10^{9}$ & 2 \\
\hline 53.16848 & -27.76772 & 1.2615 & 5.42 & 0.92 & $\mathrm{Y}$ & 0.49 & $3.71 \times 10^{9}$ & 2 \\
\hline
\end{tabular}


Table 6

(Continued)

\begin{tabular}{|c|c|c|c|c|c|c|c|c|}
\hline $\begin{array}{l}\text { R.A. } \\
\text { (deg) } \\
\text { (1) }\end{array}$ & $\begin{array}{l}\text { Decl. } \\
\text { (deg) } \\
(2)\end{array}$ & $z_{\mathrm{CO}}$ & $\begin{array}{l}\mathrm{S} / \mathrm{N} \\
(4)\end{array}$ & $\begin{array}{l}\text { Compl. } \\
\text { (5) }\end{array}$ & $\begin{array}{c}\mathrm{c} / \mathrm{p} ? \\
(6)\end{array}$ & $\begin{array}{l}\text { fid. } \\
\text { (7) }\end{array}$ & $\begin{array}{c}L^{\prime} \\
(8) \\
\left(\mathrm{K} \mathrm{km} \mathrm{s}^{-1} \mathrm{pc}^{2}\right)\end{array}$ & $J_{\text {up }}$ \\
\hline 53.16946 & -27.79258 & 0.1428 & 5.26 & 1.00 & $\mathrm{~N}$ & 0.55 & $1.03 \times 10^{8}$ & $\overline{1}$ \\
\hline 53.14334 & -27.78797 & 3.1259 & 5.25 & 1.00 & $\mathrm{Y}$ & 0.38 & $5.31 \times 10^{9}$ & 4 \\
\hline 53.14437 & -27.77806 & 0.1873 & 5.23 & 1.00 & $\mathrm{~N}$ & 0.54 & $2.80 \times 10^{8}$ & 1 \\
\hline 53.16572 & -27.79701 & 3.2263 & 5.23 & 0.76 & $\mathrm{~N}$ & 0.55 & $3.33 \times 10^{9}$ & 4 \\
\hline 53.14444 & -27.78346 & 2.2128 & 5.12 & 1.00 & $\mathrm{~N}$ & 0.55 & $5.83 \times 10^{9}$ & 3 \\
\hline 53.16161 & -27.77591 & 1.2456 & 5.09 & 1.00 & $\mathrm{~N}$ & 0.24 & $2.24 \times 10^{9}$ & 2 \\
\hline 53.17350 & -27.79211 & 1.3310 & 5.05 & 0.93 & $\mathrm{~N}$ & 0.28 & $3.61 \times 10^{9}$ & 2 \\
\hline 53.14514 & -27.79452 & 1.0398 & 4.97 & 1.00 & $\mathrm{~N}$ & 0.45 & $4.33 \times 10^{9}$ & 2 \\
\hline 53.14967 & -27.78415 & 1.5710 & 4.93 & 0.97 & $\mathrm{Y}$ & 0.39 & $3.78 \times 10^{9}$ & 2 \\
\hline 53.14690 & -27.78514 & 3.5460 & 4.92 & 1.00 & $\mathrm{~N}$ & 0.50 & $4.15 \times 10^{9}$ & 4 \\
\hline
\end{tabular}

Note. (1-2) Sky coordinates of the line candidate. (3) Adopted CO-based redshift. (4) Signal-to-noise. (5) Completeness (see GL19). (6) Does the line candidate have a counterpart at optical/nir wavelengths with matching redshift (see the text)? (7) Fidelity of the line candidate (see GL19). (8) Inferred line luminosity. (9) Rotational quantum number of the upper energy level of the transition.

(This table is available in machine-readable form.)

\section{Appendix B Robustness of the CO LFs}

Here we test the robustness of the CO LFs constraints from ASPECS LP $3 \mathrm{~mm}$ by creating different realizations of the CO LFs after altering some of the assumptions discussed in the previous section, in particular, concerning the fidelity of line candidates, and the $\mathrm{CO}$ excitation. The results of these tests are displayed in Figures 10 and 11.

\section{B.1. Impact of Uncertain Redshifts/Sources with No Counterparts}

First, we compare our CO LFs and the constraints on the $\rho\left(\mathrm{H}_{2}\right)$ evolution with redshift against the ones we infer, if we only subselect the galaxies for which a catalog redshift is available, and is consistent with the CO-based redshift within $|\delta z|<0.1$ (see Figure 5). This automatically removes all the line candidates from the line search that lack a counterpart at other wavelengths, as well as potential misassociations with foreground/background galaxies.

The inferred CO LFs are practically unaltered at their bright end. Small deviations are reported at the faint end, likely due to a combination of two reasons: (1) At the faint end, the impact of false positive candidates is larger. These spurious candidates by definition have counterparts only due to chance alignment, and it is unlikely that such counterparts have matching redshifts. (2) For reasonable ranges of the gas fraction $M_{\mathrm{H} 2} / M_{*}$, fainter $\mathrm{CO}$ lines are typically associated with fainter stellar emission; these optical/NIR-faint galaxies might have relatively large redshift uncertainties, and might get scattered out of the $|\delta z|<0.1$ selection.

The direct consequence of these discrepancies is that $\rho\left(\mathrm{H}_{2}\right)$ estimated only using sources with redshift-matching counterparts shows a faster decline at increasing redshifts at $z>3$, compared to our reference estimate, although the two estimates are well within $1 \sigma$ uncertainties in both the CO LFs and $\rho\left(\mathrm{H}_{2}\right)$ at any redshift. We thus conclude that our results, and in particular the steep evolution in $\rho\left(\mathrm{H}_{2}\right)$ from present day to $z \gtrsim 1$, are not significantly affected by our treatment of sources without clear counterparts or with ambiguous redshift associations.

\section{B.2. Impact of $\mathrm{CO}$ Excitation}

We then examine the impact of the $\mathrm{CO}$ excitation assumptions on our estimates of the $\mathrm{CO}(1-0) \mathrm{LFs}$ and on $\rho\left(\mathrm{H}_{2}\right)$ (the CO LFs of the observed transitions are naturally unaffected by this assumption). We do so by repeating our analysis after assuming two extreme cases: a high excitation case corresponding to thermalized $\mathrm{CO}$ up to $J_{\mathrm{up}}=4$, and a low-excitation scenario where the $\mathrm{CO}$ emission is modeled based on the Milky Way disk (see, e.g., Weiß et al. 2007; Carilli \& Walter 2013). A higher (lower) excitation implies fainter (brighter) $L_{\mathrm{CO}(1-0)}^{\prime}$ for a given line observed in a $J_{\text {up }}>1$ transition, and therefore lower (higher) values of $M_{\mathrm{H} 2}$. For reference, our fiducial assumption based on Daddi et al. (2015) lies roughly half way between these two extreme cases for the transitions of interest here.

We find that a thermalized $\mathrm{CO}$ scenario would mitigate, but not completely solve, the friction between the ASPECS LP $3 \mathrm{~mm}$ CO LFs and the predictions by semianalytical models. This is further explored in Popping et al. (2019). A lowexcitation scenario, on the other hand, would exacerbate the tension. Evidence of a strong evolution in $\rho\left(\mathrm{H}_{2}\right)$ between the local universe and $z>1$ is confirmed irrespective of the assumptions on the $\mathrm{CO}$ excitation, but for a low-excitation scenario, $\rho\left(\mathrm{H}_{2}\right)$ appears nearly constant at any $z>1$, while it would drop rapidly at increasing redshifts, if a thermalized $\mathrm{CO}$ excitation is assumed. 

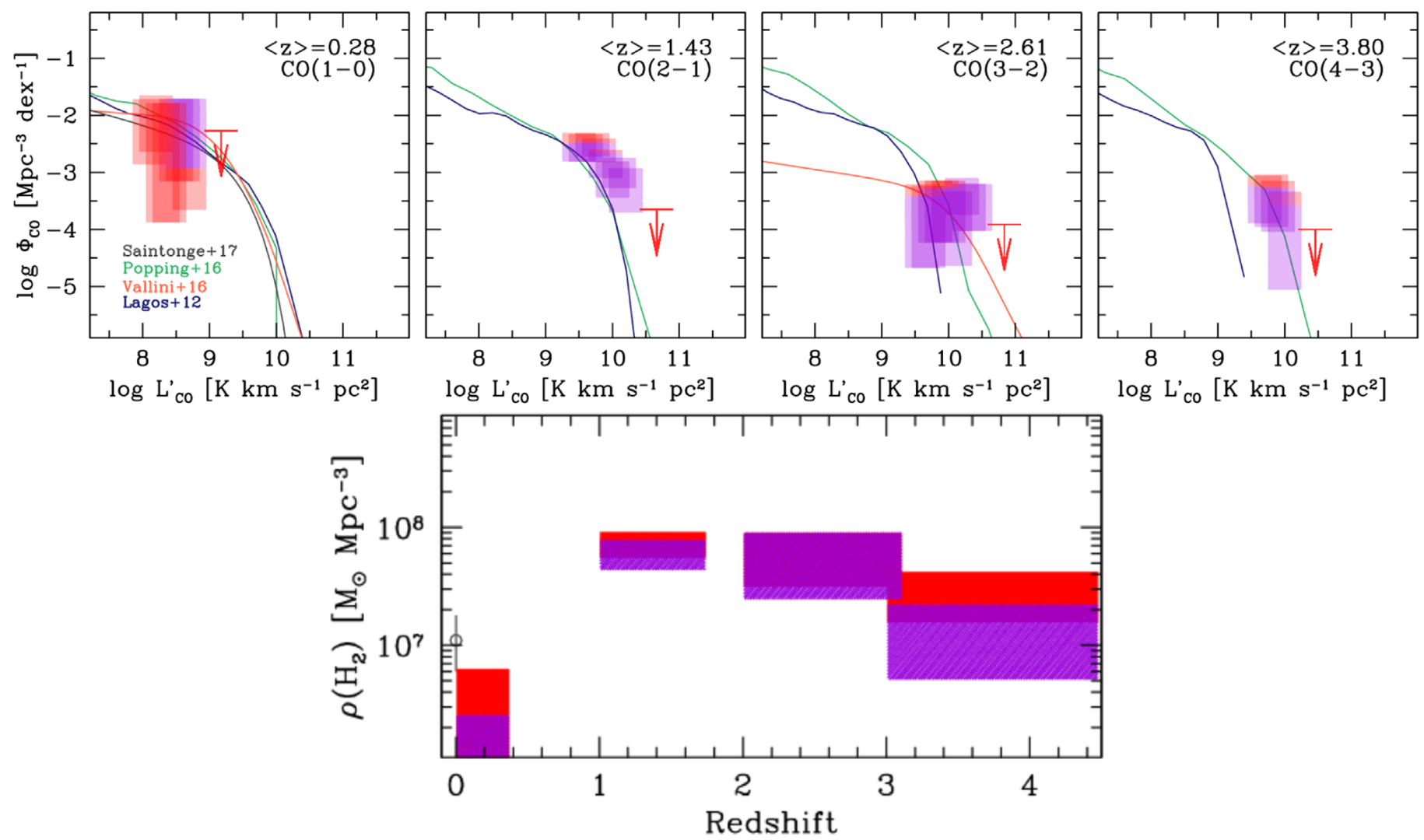

Figure 10. CO LFs and evolution of $\rho\left(\mathrm{H}_{2}\right)$ with redshift derived from the entire sample (red shaded boxes) and from the subsample of line candidates that present a counterpart with matching redshifts $(|\delta z|<0.1)$. The vertical extent of each box marks the $1 \sigma$ confidence range. Empirical and semianalytical model predictions are shown for reference. All of the CO luminosity functions appear consistent in the bright end; at lower luminosities (below $L_{\mathrm{CO}}^{\prime} \approx 10^{10} \mathrm{~K} \mathrm{~km} \mathrm{~s}{ }^{-1} \mathrm{pc}^{2}$ ) discrepancies arise due to the combined effect of larger rate of false positive candidates at the faint end, and intrinsically fainter counterparts. Our estimates of $\rho\left(\mathrm{H}_{2}\right)$ also appear relatively unchanged if we only focus on sources with matching redshifts, at least up to $z \sim 3$. 

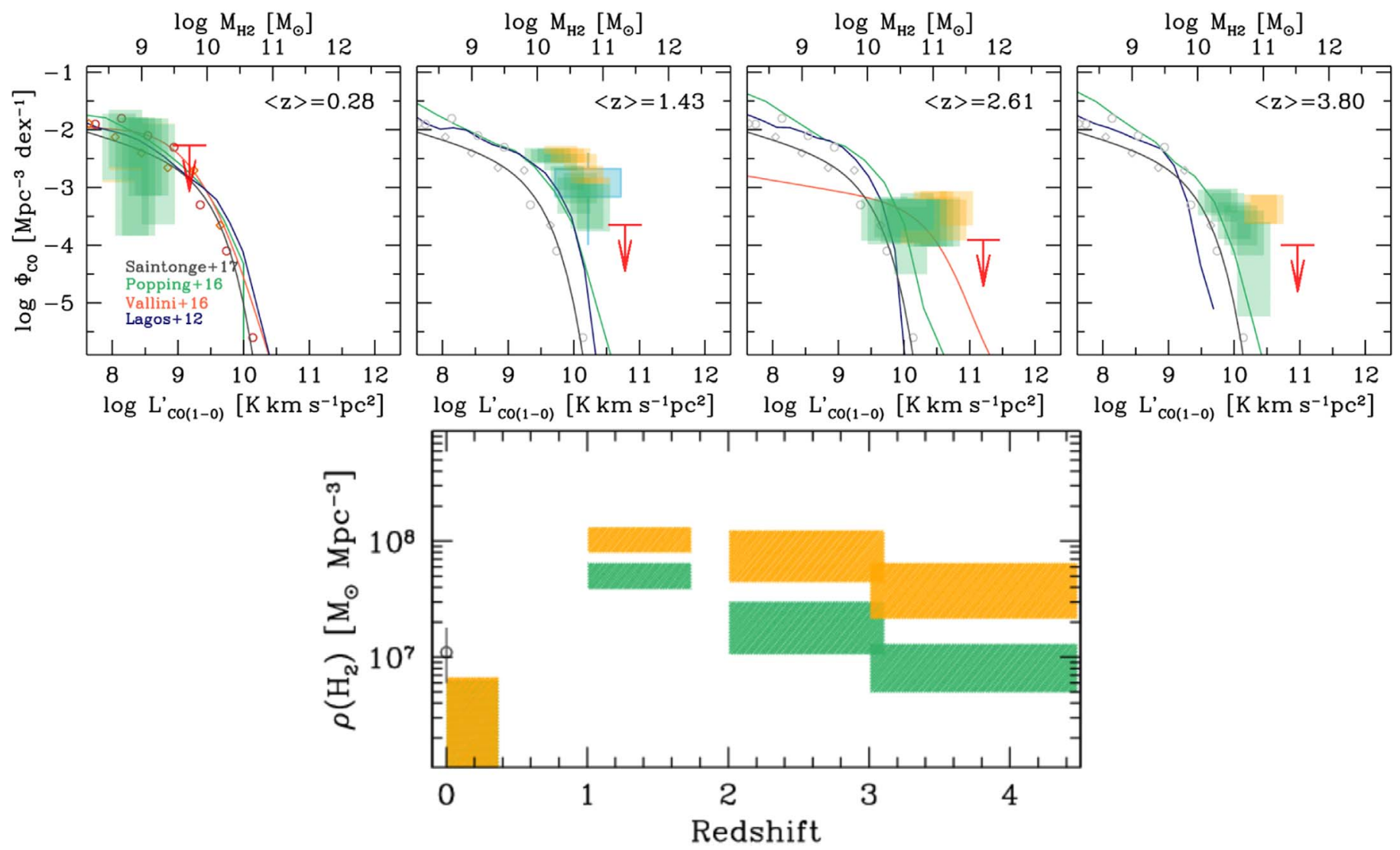

Figure 11. $\mathrm{CO}(1-0) \mathrm{LFs}$ and evolution of $\rho\left(\mathrm{H}_{2}\right)$ with redshift derived assuming two extreme cases of maximal (=thermalized) and minimal (=Milky Way-like) $\mathrm{CO}$ excitation, shown in green and orange, respectively. The Milky Way excitation is taken from Weiß et al. (2007). The thermalized case assumes $r_{J 1}=J^{2}$ for all the CO transitions. The vertical extent of the boxes marks the $1 \sigma$ confidence intervals. The Daddi et al. (2015) CO excitation adopted elsewhere in this paper lies in between these two extreme cases. A high excitation scenario would be in better agreement with the semianalytical models, especially at the bright end, although it would not be enough to fully account for the discrepancy, especially at $z=1-3$. A high excitation model would also slightly reduce the evolution in $\rho\left(\mathrm{H}_{2}\right)$ between $z=1-3$ and the local universe (by a factor $<2$ with respect to our fiducial assumption), and would naturally predict a faster drop of $\rho\left(\mathrm{H}_{2}\right)$ toward high redshifts.

\section{ORCID iDs}

Roberto Decarli (i) https://orcid.org/0000-0002-2662-8803 Fabian Walter (1) https://orcid.org/0000-0003-4793-7880 Manuel Aravena (10) https://orcid.org/0000-0002-6290-3198 Leindert Boogaard (1) https://orcid.org/0000-0002-3952-8588 Chris Carilli (1) https://orcid.org/0000-0001-6647-3861 Emanuele Daddi (1) https://orcid.org/0000-0002-3331-9590 Gergö Popping (1) https://orcid.org/0000-0003-1151-4659 Dominik Riechers (i) https://orcid.org/0000-0001-9585-1462 Bade Uzgil (1) https://orcid.org/0000-0001-8526-3464 Axel Weiss (10) https://orcid.org/0000-0003-4678-3939 Roberto J. Assef (10 https://orcid.org/0000-0002-9508-3667 Franz Erik Bauer (1) https://orcid.org/0000-0002-8686-8737 Frank Bertoldi (1) https://orcid.org/0000-0002-1707-1775 Rychard Bouwens (1) https://orcid.org/0000-0002-4989-2471 Thierry Contini (1) https://orcid.org/0000-0003-0275-938X Paulo C. Cortes (i) https://orcid.org/0000-0002-3583-780X Jacqueline Hodge (i) https://orcid.org/0000-0001-6586-8845 Rob Ivison (10) https://orcid.org/0000-0001-5118-1313 Benjamin Magnelli (i) https://orcid.org/0000-0002-6777-6490 Mladen Novak 수 https://orcid.org/0000-0001-8695-825X Pascal Oesch 주 https://orcid.org/0000-0001-5851-6649 Hans-Walter Rix (1) https://orcid.org/0000-0003-49969069 난 https://orcid.org/0000-0003-4996-9069 Mark T. Sargent (1) https://orcid.org/0000-0003-1033-9684 Ian Smail ๑ https://orcid.org/0000-0003-3037-257X A. Mark Swinbank (i) https://orcid.org/0000-0003-1192-5837

\section{References}

Aravena, M., Carilli, C. L., Salvato, M., et al. 2012, MNRAS, 426, 258 Aravena, M., Decarli, R., Walter, F., et al. 2016a, ApJ, 833, 68 Aravena, M., Decarli, R., Walter, F., et al. 2016b, ApJ, 833, 71 Aravena, M., Spilker, J. S., Bethermin, M., et al. 2016c, MNRAS, 457, 4406

Aravena, M., Decarli, R., Gonzalez-Lopez, J., et al. 2019, ApJ, 882, 136 Ashby, M. L. N., Willner, S. P., Fazio, G. G., et al. 2013, ApJ, 769, 80 Bacon, R., Conseil, S., Mary, D., et al. 2017, A\&A, 608, A1 Beckwith, S. V., Stiavelli, M., Koekemoer, A. M., et al. 2006, AJ, 132, 1729 Bolatto, A. D., Wolfire, M., \& Leroy, A. K. 2013, ARA\&A, 51, 207 Boogaard, L., Decarli, R., Lopez-Gonzalez, J., et al. 2019, ApJ, 882, 140 Boselli, A., Cortese, L., Boquien, M., et al. 2014, A\&A, 564, A66 Bouwens, R. J., Aravena, M., Decarli, R., et al. 2016, ApJ, 833, 72 Bouwens, R. J., Illingworth, G. D., Oesch, P. A., et al. 2014, ApJ, 793, 115 Bouwens, R. J., Illingworth, G. D., Oesch, P. A., et al. 2015, ApJ, 803, 34 Cardamone, C. N., van Dokkum, P. G., Urry, C. M., et al. 2010, ApJS, 189,270

Carilli, C. L., Chluba, J., Decarli, R., et al. 2016, ApJ, 833, 73

Carilli, C. L., \& Walter, F. 2013, ARA\&A, 51, 105

Chabrier, G. 2003, PASP, 115, 763

Chapman, S. C., Bertoldi, F., Smail, I., et al. 2015, MNRAS, 449, L68

Coe, D., Benítez, N., Sánchez, S. F., et al. 2006, AJ, 132, 926 da Cunha, E., Charlot, S., \& Elbaz, D. 2008, MNRAS, 388, 1595 da Cunha, E., Groves, B., Walter, F., et al. 2013, ApJ, 766, 13 da Cunha, E., Walter, F., Smail, I. R., et al. 2015, ApJ, 806, 110 Daddi, E., Bournaud, F., Walter, F., et al. 2010a, ApJ, 713, 686 Daddi, E., Dannerbauer, H., Liu, D., et al. 2015, A\&A, 577, 46 Daddi, E., Elbaz, D., Walter, F., et al. 2010b, ApJL, 714, L118 Decarli, R., Walter, F., Aravena, M., et al. 2016a, ApJ, 833, 69 Decarli, R., Walter, F., Aravena, M., et al. 2016b, ApJ, 833, 70 Decarli, R., Walter, F., Carilli, C., et al. 2014, ApJ, 782, 78 
Dessauges-Zavadsky, M., Zamojski, M., Rujopakarn, W., et al. 2017, A\&A, 605, A81

Dessauges-Zavadsky, M., Zamojski, M., Schaerer, D., et al. 2015, A\&A, 577, A50

Dickinson, M., Papovich, C., Ferguson, H. C., \& Budavári, T. 2003, ApJ, 587,25

Dunlop, J. S., McLure, R. J., Biggs, A. D., et al. 2017, MNRAS, 466, 861

Elbaz, D., Dickinson, M., Hwang, H. S., et al. 2011, A\&A, 533, 119

Erben, T., Schirmer, M., Dietrich, J. P., et al. 2005, AN, 326, 432

Genzel, R., Newman, S., Jones, T., et al. 2011, ApJ, 733, 101

Genzel, R., Tacconi, L. J., Gracia-Carpio, J., et al. 2010, MNRAS, 407, 2091

Genzel, R., Tacconi, L. J., Lutz, D., et al. 2015, ApJ, 800, 20

Giavalisco, M., Ferguson, H. C., Koekemoer, A. M., et al. 2004, ApJL, 600, L93

González-López, J., Barrientos, L. F., Gladders, M. D., et al. 2017, ApJL, 846, L22

González-López, J., et al. 2019, ApJ, 882, 139

Grogin, N. A., Kocevski, D. D., Faber, S. M., et al. 2011, ApJS, 197, 35

Gruppioni, C., Pozzi, F., Rodighiero, G., et al. 2013, MNRAS, 432, 23

Harris, A. I., Baker, A. J., Frayer, D. T., et al. 2012, ApJ, 752, 152

Hayashi, M., Tadaki, K., Kodama, T., et al. 2018, ApJ, 856, 118

Hayatsu, N. H., Matsuda, Y., Umehata, H., et al. 2017, PASJ, 69, 45

Hildebrandt, H., Erben, T., Dietrich, J. P., et al. 2006, A\&A, 452, 1121

Hsieh, B.-C., Wang, W.-H., Hsieh, C.-C., et al. 2012, ApJS, 203, 23

Ilbert, O., McCracken, H. J., Le Fèvre, O., et al. 2013, A\&A, 556, 55

Illingworth, G. D., Magee, D., Oesch, P. A., et al. 2013, ApJS, 209, 6

Inami, H., Bacon, R., Brinchmann, J., et al. 2017, A\&A, 608, A2

Keating, G. K., Marrone, D. P., Bower, G. C., et al. 2016, ApJ, 830, 34

Kennicutt, R. C., \& Evans, N. J. 2012, ARA\&A, 50, 531

Keres, D., Yun, M. S., \& Young, J. S. 2003, ApJ, 582, 659

Koekemoer, A. M., Ellis, R. S., McLure, R. J., et al. 2013, ApJS, 209, 3

Koekemoer, A. M., Faber, S. M., Ferguson, H. C., et al. 2011, ApJS, 197, 36

Lagos, C. d. P., Baugh, C. M., Lacey, C. G., et al. 2011, MNRAS, 418, 1649

Lagos, C. d. P., Bayet, E., Baugh, C. M., et al. 2012, MNRAS, 426, 2142

Le Fèvre, O., Vettolani, G., Garilli, B., et al. 2005, A\&A, 439, 845

Lee, M. M., Tanaka, I., Kawabe, R., et al. 2017, ApJ, 842, 55

Leipski, C., Meisenheimer, K., Walter, F., et al. 2014, ApJ, 785, 154

Lentati, L., Wagg, J., Carilli, C. L., et al. 2015, ApJ, 800, 67

Luo, B., Brandt, W. N., Xue, Y. Q., et al. 2017, ApJS, 228, 2

Madau, P., \& Dickinson, M. 2014, ARA\&A, 52, 415

Magnelli, B., Lutz, D., Saintonge, A., et al. 2014, A\&A, 561, A86

Magnelli, B., Popesso, P., Berta, S., et al. 2013, A\&A, 553, 132

Magnelli, B., Saintonge, A., Lutz, D., et al. 2012, A\&A, 548, 22

Marrone, D. P., Spilker, J. S., Hayward, C. C., et al. 2018, Natur, 553, 51

McLure, R. J., Dunlop, J. S., Bowler, R. A. A., et al. 2013, MNRAS, 432, 2696

McMullin, J. P., Waters, B., Schiebel, D., Young, W., \& Golap, K. 2007, in ASP Conf. Ser. 376, Astronomical Data Analysis Software and Systems XVI, ed. R. A. Shaw, F. Hill, \& D. J. Bell (San Francisco, CA: ASP), 127

Miller, T. B., Chapman, S. C., Aravena, M., et al. 2018, Natur, 556, 469

Momcheva, I. G., Brammer, G. B., van Dokkum, P. G., et al. 2016, ApJS, 225,27

Morris, A. M., Kocevski, D. D., Trump, J. R., et al. 2015, AJ, 149, 178
Noble, A. G., McDonald, M., Muzzin, A., et al. 2017, ApJL, 842, L21

Noeske, K. G., Weiner, B. J., Faber, S. M., et al. 2007, ApJL, 660, L43

Nonino, M., Dickinson, M., Rosati, P., et al. 2009, ApJS, 183, 244

Obreschkow, D., Heywood, I., Klöckner, H.-R., \& Rawlings, S. 2009, ApJ, 702,1321

Obreschkow, D., \& Rawlings, S. 2009, ApJL, 696, L129

Omont, A. 2007, RPPh, 70, 1099

Oteo, I., Ivison, R. J., Dunne, L., et al. 2018, ApJ, 856, 72

Pappovich, C., Labbé, I, Glazebrook, K., et al. 2016, NatAs, 1, 3

Pavesi, R., Sharon, C. E., Riechers, D. A., et al. 2018, ApJ, 864, 49

Planck Collaboration, Ade, P. A. R., Aghanim, N., et al. 2016, A\&A, 594, A13

Popping, G., Somerville, R. S., \& Trager, S. C. 2014, MNRAS, 442, 2398

Popping, G., van Kampen, E., Decarli, R., et al. 2016, MNRAS, 461, 93

Popping, G., Pillepich, A., Somerville, R. S., et al. 2019, ApJ, 882, 137

Retzlaff, J., Rosati, P., Dickinson, M., et al. 2010, A\&A, 511, A50

Rhoads, J. E., Malhotra, S., Pirzkal, N., et al. 2009, ApJ, 697, 942

Riechers, D. A., Bradford, C. M., Clements, D. L., et al. 2013, Natur, 496, 329

Riechers, D. A., Carilli, C. L., Walter, F., \& Momjian, E. 2010, ApJL, 724, L153

Riechers, D. A., Pavesi, R., Sharon, C. E., et al. 2019, ApJ, 872, 7

Rudnick, G., Hodge, J., Walter, F., et al. 2017, ApJ, 849, 27

Rujopakarn, W., Dunlop, J. S., Rieke, G. H., et al. 2016, ApJ, 833, 12

Saintonge, A., Catinella, B., Tacconi, L. J., et al. 2017, ApJS, 233, 22

Sargent, M. T., Daddi, E., Béthermin, M., et al. 2014, ApJ, 793, 19

Schechter, P. 1976, ApJ, 203, 297

Schenker, M. A., Robertson, B. E., Ellis, R. S., et al. 2013, ApJ, 768, 196

Schinnerer, E., Groves, B., Sargent, M. T., et al. 2016, ApJ, 833, 112

Scoville, N., Abraham, R. G., Aussel, H., et al. 2007, ApJS, 172, 1

Scoville, N., Lee, N., Vanden Bout, P., et al. 2017, ApJ, 837, 150

Seko, A., Ohta, K., Yabe, K., et al. 2016, ApJ, 819, 82

Silverman, J. D., Daddi, E., Rodighiero, G., et al. 2015, ApJL, 812, L23

Silverman, J. D., Rujopakarn, W., Daddi, E., et al. 2018, ApJ, 867, 92

Skelton, R. E., Whitaker, K. E., Momcheva, I. G., et al. 2014, ApJS, 214, 24

Strandet, M. L., Weiss, A., De Breuck, C., et al. 2017, ApJL, 842, L15

Tacconi, L. J., Genzel, R., Neri, R., et al. 2010, Natur, 463, 781

Tacconi, L. J., Genzel, R., Saintonge, A., et al. 2018, ApJ, 853, 179

Tacconi, L. J., Neri, R., Genzel, R., et al. 2013, ApJ, 768, 74

Tunnard, R., \& Greve, T. R. 2016, ApJ, 819, 161

Vallini, L., Gruppioni, C., Pozzi, F., Vignali, C., \& Zamorani, G. 2016, MNRAS, 456, L40

van der Wel, A., Bell, E. F., Häussler, B., et al. 2012, ApJS, 203, 24

Venemans, B. P., Walter, F., Decarli, R., et al. 2017, ApJ, 845, 154

Walter, F., Decarli, R., Aravena, M., et al. 2016, ApJ, 833, 67

Walter, F., Decarli, R., Carilli, C., et al. 2012, Natur, 486, 233

Walter, F., Decarli, R., Sargent, M., et al. 2014, ApJ, 782, 79

Weiß, A., Downes, D., Neri, R., et al. 2007, A\&A, 467, 955

Whitaker, K. E., Franx, M., Leja, J., et al. 2014, ApJ, 795, 104

Williams, R. E., Blacker, B., Dickinson, M., et al. 1996, AJ, 112, 1335

Wuyts, S., Labbé, I., Förster Schreiber, N. M., et al. 2008, ApJ, 682, 985

Xie, L., De Lucia, G., Hirschmann, M., Fontanot, F., \& Zoldan, A. 2017, MNRAS, 469, 968

Xu, C., Pirzkal, N., Malhotra, S., et al. 2007, AJ, 134, 169 